This article has been published in a revised form in Publications of the Astronomical Society of Australia [http://doi.org/10.1017/pasa.2019.16]. This version is free to view and download for private research and study only. Not for re-distribution or re-use. @ Astronomical Society of Australia 2019.

\title{
ASKAP Commissioning Observations of the GAMA 23 Field
}

Denis A. Leahy ${ }^{1 *}$, A.M. Hopkins ${ }^{2}$, R. P. Norris ${ }^{3,4}$, J. Marvil ${ }^{4,5}$, J.D. Collier ${ }^{3,4,26}$, E.N. Taylor ${ }^{6}$, J.R. Allison $^{7}$, C. Anderson ${ }^{4}$, M. Bell ${ }^{8}$, M. Bilicki ${ }^{9,10}$, J. Bland-Hawthorn ${ }^{11}$, S. Brough ${ }^{12}$, M.J.I. Brown ${ }^{13}$, S. Driver ${ }^{14}$, G. Gurkan ${ }^{15}$, L. Harvey-Smith ${ }^{4,3}$, I. Heywood ${ }^{16,17}$, B.W. Holwerda ${ }^{18}$, J. Liske ${ }^{19}$, A. R. Lopez-Sanchez ${ }^{2}$, D. McConnell ${ }^{4}$, A. Moffett ${ }^{20}$, M.S. Owers ${ }^{21}$, K.A. Pimbblet ${ }^{22}$, W. Raja ${ }^{4}$, N. Seymour $^{23}$, M.A. Voronkov ${ }^{4}$, and L. Wang ${ }^{24,25}$

${ }^{1}$ Department of Physics and Astronomy, University of Calgary, Calgary, Alberta, T2N 1N4, Canada

${ }^{2}$ Australian Astronomical Optics, Macquarie University,105 Delhi Rd, North Ryde, NSW 2113, Australia

${ }^{3}$ Western Sydney University, Locked Bag 1797, Penrith, NSW, 2751, Australia

${ }^{4}$ CSIRO Astronomy and Space Science, Australia Telescope National Facility, PO Box 76, Epping, NSW 1710, Australia

${ }^{5}$ National Radio Astronomical Observatory, P.O. Box O, 1003 Lopezville Road, Socorro, NM 87801-0387, USA

${ }^{6}$ Centre for Astrophysics and Supercomputing, Swinburne University of Technology, Hawthorn, Vic 3122, Australia

${ }^{7}$ Sub-Dept. of Astrophysics, Department of Physics, University of Oxford, Denys Wilkinson Building, Keble Rd., Oxford, OX1 3RH, UK

${ }^{8}$ University of Technology Sydney, 15 Broadway, Ultimo NSW 2007, Australia

${ }^{9}$ Leiden Observatory, Leiden University, P.O. Box 9513, NL-2300 RA Leiden, The Netherlands

${ }^{10}$ National Centre for Nuclear Research, Astrophysics Division, P.O. Box 447, PL-90-950 Łódź, Poland

${ }^{11}$ Sydney Institute for Astronomy, School of Physics A28, University of Sydney, NSW 2006, Australia

${ }^{12}$ School of Physics, University of New South Wales, NSW 2052, Australia

${ }^{13}$ School of Physics and Astronomy, Monash University, Clayton, VIC 3800, Australia

${ }^{14}$ International Centre for Radio Astronomy Research (ICRAR), University of Western Australia, Stirling Highway, Perth, Western Australia

${ }^{15}$ CSIRO Astronomy and Space Science, PO Box 1130, Bentley WA 6102, Australia

${ }^{16}$ Astrophysics, Department of Physics, University of Oxford, Keble Road, Oxford OX1 3RH, UK

${ }^{17}$ Department of Physics and Electronics, Rhodes University, PO Box 94, Grahamstown, 6140, South Africa

${ }^{18}$ Department of Physics and Astronomy, 102 Natural Science Building, University of Louisville, Louisville KY 40292, USA

${ }^{19}$ Hamburger Sternwarte, Universitat Hamburg, Gojenbergsweg 112, 21029 Hamburg, Germany

${ }^{20}$ Department of Physics and Astronomy, Vanderbilt University, USA

${ }^{21}$ Department of Physics and Astronomy, Macquarie University, NSW 2109, Australia

${ }^{22}$ E.A.Milne Centre for Astrophysics, University of Hull, Cottingham Road, Kingston-upon-Hull, HU6 7RX, UK

${ }^{23}$ International Centre for Radio Astronomy Research, Curtin University, Bentley WA 6102, Australia

${ }^{24}$ SRON Netherlands Institute for Space Research, Landleven 12, 9747 AD, Groningen, The Netherlands

${ }^{25}$ Kapteyn Astronomical Institute, University of Groningen, Postbus 800, $9700 \mathrm{AV}$, Groningen, The Netherlands

${ }^{26}$ The Inter-University Institute for Data Intensive Astronomy (IDIA), Department of Astronomy, University of Cape Town,

Rondebosch, 7701, South Africa

\begin{abstract}
We have observed the G23 field of the Galaxy And Mass Assembly (GAMA) survey using the Australian Square Kilometre Array Pathfinder (ASKAP) in its commissioning phase, to validate the performance of the telescope and to characterize the detected galaxy populations. This observation covers $\sim 48 \mathrm{deg}^{2}$ with synthesized beam of $32.7^{\prime \prime}$ by $17.8^{\prime \prime}$ at $936 \mathrm{MHz}$, and $\sim 39 \mathrm{deg}^{2}$ with synthesized beam of $15.8^{\prime \prime}$ by $12.0^{\prime \prime}$ at $1320 \mathrm{MHz}$. At both frequencies, the r.m.s. (root-mean-square) noise is $\sim 0.1 \mathrm{mJy} / \mathrm{beam}$. We combine these radio observations with the GAMA galaxy data, which includes spectroscopy of galaxies that are i-band selected with a magnitude limit of 19.2. Wide-field Infrared Survey Explorer (WISE) infrared (IR) photometry is used to determine which galaxies host an active galactic nucleus (AGN). In properties including source counts, mass distributions, and IR vs. radio luminosity relation, the ASKAP detected radio sources behave as expected. Radio galaxies have higher stellar mass and luminosity in IR, optical and UV than other galaxies. We apply optical and IR AGN diagnostics and find that they disagree for $\sim 30 \%$ of the galaxies in our sample. We suggest possible causes for the disagreement. Some cases can be explained by optical extinction of the AGN, but for more than half of the cases we do not find a clear explanation. Radio sources are more likely $(\sim 6 \%)$ to have an AGN than radio quiet galaxies $(\sim 1 \%)$, but the majority of AGN are not detected in radio at this sensitivity.
\end{abstract}

Keywords: radio continuum: galaxies - surveys - infrared: galaxies - galaxies: active - galaxies: statistics 


\section{INTRODUCTION}

The moderate redshift Universe $(0.1 \lesssim z \lesssim 1)$ has in the past decade become accessible for large surveys of galaxies at optical and IR wavelengths. However, most radio surveys have sampled different populations from these, mainly because most bright radio sources represent the synchrotron emission from active galactic nuclei (AGN) (e.g. Heckman \& Best 2014), which are relatively uncommon in optical and IR surveys. This is now changing, as the largest area radio surveys are now reaching the sensitivity where they are dominated by low-redshift star forming galaxies (SFG) (e.g. Norris 2017 ), in which the radio emission is powered by star formation processes. Thus, it is an opportune time to compare radio and galaxy surveys to find the same objects emitting in radio and optical/IR bands.

The Evolutionary Map of the Universe (EMU) (Norris et al., 2011) is a wide-field radio continuum survey planned for the new Australian Square Kilometre Array Pathfinder (ASKAP) telescope (Johnston et al., 2008). The primary goal of EMU is to make a deep (r.m.s. $\sim 10 \mu \mathrm{Jy} /$ beam) radio continuum survey of the entire Southern sky at $1.3 \mathrm{GHz}$, extending as far North as +30 declination, with a resolution of 10 arcsec. EMU is expected to detect and catalogue about 70 million galaxies, including typical SFG up to $\mathrm{z} \sim 1$, powerful starbursts to even greater redshifts, and AGN to the edge of the visible Universe. Here we report early EMU observations, taken during the commissioning phase of ASKAP, with the goal of characterizing the galaxy and radio galaxy samples.

Radio galaxies can be divided into two classes: SFG and AGN. The radio emission from SFG is synchrotron emission of cosmic-ray electrons accelerated by shocks from supernovae, and so the radio emission is confined to the inner few kpc of a galaxy, and is seen in the radio image as a single unresolved component. The radio emission is relatively weak, is strongly correlated with the far-IR flux, and is typically detected from galaxies in the nearby Universe, with a median redshift $z<0.5$. In AGN, the radio emission is synchrotron emission emitted by the jets and lobes of relativistic electrons accelerated from the environs of a supermassive black hole, and may appear as a single, double, or triple component, or sometimes as a complex structure with visible jets. The radio emission can be very strong, and easily detectable up to very high redshifts.

Although these two types of radio source are powered by very different physical mechanisms, it is notoriously hard to distinguish between them from the radio data alone (Norris et al., 2013). While multiple-component sources and strong high-redshift sources are probably AGN, a single component source at low redshift, or with no measured redshift, may be either AGN or SFG.

\footnotetext{
*E-mail: leahy@ucalgary.ca
}

Optical line ratios have been used as a diagnostic to classify galaxies as SFG or AGN since it was proposed by Baldwin et al. (1981). We use this BaldwinPhillips-Terlevich (BPT) diagnostic in the form developed by Kewley et al. (2001), which uses the line ratios [OIII] $\lambda 5007 / H \beta$ and [SII] $\lambda \lambda 6717,6731 / H \alpha$ (e.g., Hopkins et al., 2013). The line ratios are sensitive to the spectral hardness of the radiation which ionizes the gas, and can be used to determine when the radiation is too spectrally hard to originate from star formation, i.e., the ionizing radiation can only be produced by an AGN. We refer to the $([\mathrm{OIII}] \lambda 5007 / H \beta)$ vs $([\mathrm{SII}] \lambda \lambda 6717,6731 / H \alpha)$ diagnostic as the BPT diagram and refer to this set of lines as the BPT lines.

AGN generate thermal radiation in the accretion disk surrounding a supermassive black hole. Much of this is reradiated in IR by dust with a wide range of temperatures, resulting in characteristic red mid-IR colours which can be distinguished from the mid-IR colours resulting from stars. The WISE mid-IR colour criterion W1-W2 $>0.8$ was introduced by Jarrett et al. (2011), and is used here to aid in distinguishing AGN from other galaxies.

In this paper we present radio data obtained with ASKAP during its commissioning phase. One main emphasis is to verify that the radio observations are detecting real radio sources rather than imaging artifacts. The second is to use the radio data, in conjunction with optical and IR data, to study galaxy populations.

The optical data used here is from the GAMA survey (Driver et al., 2011), and the IR data from the WISE all-sky survey (Wright et al., 2010). GAMA is a spectroscopic survey of galaxies at low to moderate redshift $(z \lesssim 1)$. The GAMA regions of the sky are accessible to EMU, thus GAMA is ideal for providing optical data on the galaxies observed by EMU. With the combined radio, optical and IR data of the galaxy surveys, we investigate their masses, redshifts, and states of activity (SFG vs AGN). Our goal is to measure properties which distinguish different galactic populations, using the unique combination of depth and sky coverage of ASKAP.

This paper is organized as follows. The ASKAP radio data analysis, GAMA optical data and WISE IR data are described in Section 2. In Section 3, we identify optical and IR counterparts to the radio sources and describe the statistical properties of the various sets, including redshifts and stellar masses derived from the optical data. In Section 3.3, we apply optical and IR AGN diagnostics to classify galaxies and radio sources and compare redshift, mass and luminosity distributions of the different subsets. The properties of the radio sources and of the differences in AGN classification using optical and IR methods are discussed in Section 4. A summary of the main results is given in Section 5 .

We calculated luminosity distance from redshift, using 
standard cosmological parameters: flat universe with $H_{0}=70 \mathrm{~km} / \mathrm{s} / \mathrm{Mpc}, \Omega_{m}=0.27, \Omega_{\Lambda}=0.73$. Stellar masses were determined using a Chabrier (2003) stellar initial mass function.

\section{OBSERVATIONS AND DATA ANALYSIS}

\subsection{Radio data}

The Australian Square Kilometre Array Pathfinder (ASKAP; Johnston et al., 2008) is a new radio telescope approaching completion in Western Australia. It has a maximum baseline of $6 \mathrm{~km}$, and operates with a bandwidth of $\simeq 300 \mathrm{MHz}$ over the frequency range 700 to $1800 \mathrm{MHz}$. Each of the 36 antennas is equipped with a Phased Array Feed (PAF; Schinckel et al., 2012) giving it a field of view of $\sim 35 \mathrm{deg}^{2}$, resulting in a high survey speed. ASKAP's continuum survey is the Evolutionary Map of the Universe (EMU Norris et al., 2011), which is expected to generate a catalogue of about 70 million galaxies at $1100 \mathrm{MHz}$.

At the time of the observations described here, the telescope was in a commissioning phase (McConnell et al., 2016). Only 12 of the antennas were used, with a maximum baseline of $2 \mathrm{~km}$. An observation of PKS $B 1934$ - 638 was performed immediately adjacent in time to each observation of the target field for purposes of instrumental calibration. Each calibration observation contained one calibrator scan of duration $\sim 5$ minutes at the center of each of the 36 PAF beams. Using these data, the ASKAPsoft task "cbpcalibrator" was run to solve for the instrumental bandpass of each beam and to set the initial flux density scale based on the model of Reynolds (1994). As is customary with ASKAP, a phase reference calibrator was not included in the schedule due to the large overhead that would be incurred by observing it at the center of each beam. Instead, initial phases were transferred from the observation of the bandpass calibrator and further refined using self-calibration of the target field, and the astrometry was adjusted to match an external reference catalogue.

The observing parameters are listed in Table 1 . The PAF beam configuration was 36 beams in a $6 \times 6$ square, with two observations $\mathrm{A}$ and $\mathrm{B}$ offset by half a beam width, at a position angle of $45^{\circ}$. The $936 \mathrm{MHz}$ map covers a larger region $\left(\sim 7.3^{\circ}\right.$ by $\left.\sim 6.8^{\circ}\right)$ than the $1320 \mathrm{MHz}$ image $\left(\sim 6.6^{\circ}\right.$ by $\left.\sim 6.1^{\circ}\right)$ because of the larger primary beam size at $936 \mathrm{MHz}$. The data were reduced in ASKAPsoft using W-snapshots (Cornwell et al., 2012), which is a hybrid wide-field algorithm combining $\mathrm{W}$-projection and warped snapshot imaging. The image was cleaned (deconvolved) using the Cotton-Schwab algorithm and a single (delta function) spatial scale.

The resulting synthesized beam was $32.7^{\prime \prime}$ by $17.8^{\prime \prime}$ at $936 \mathrm{MHz}$ and $15.8^{\prime \prime}$ by $12.0^{\prime \prime}$ at $1320 \mathrm{MHz}$. The synthesized beam of the higher frequency observations is
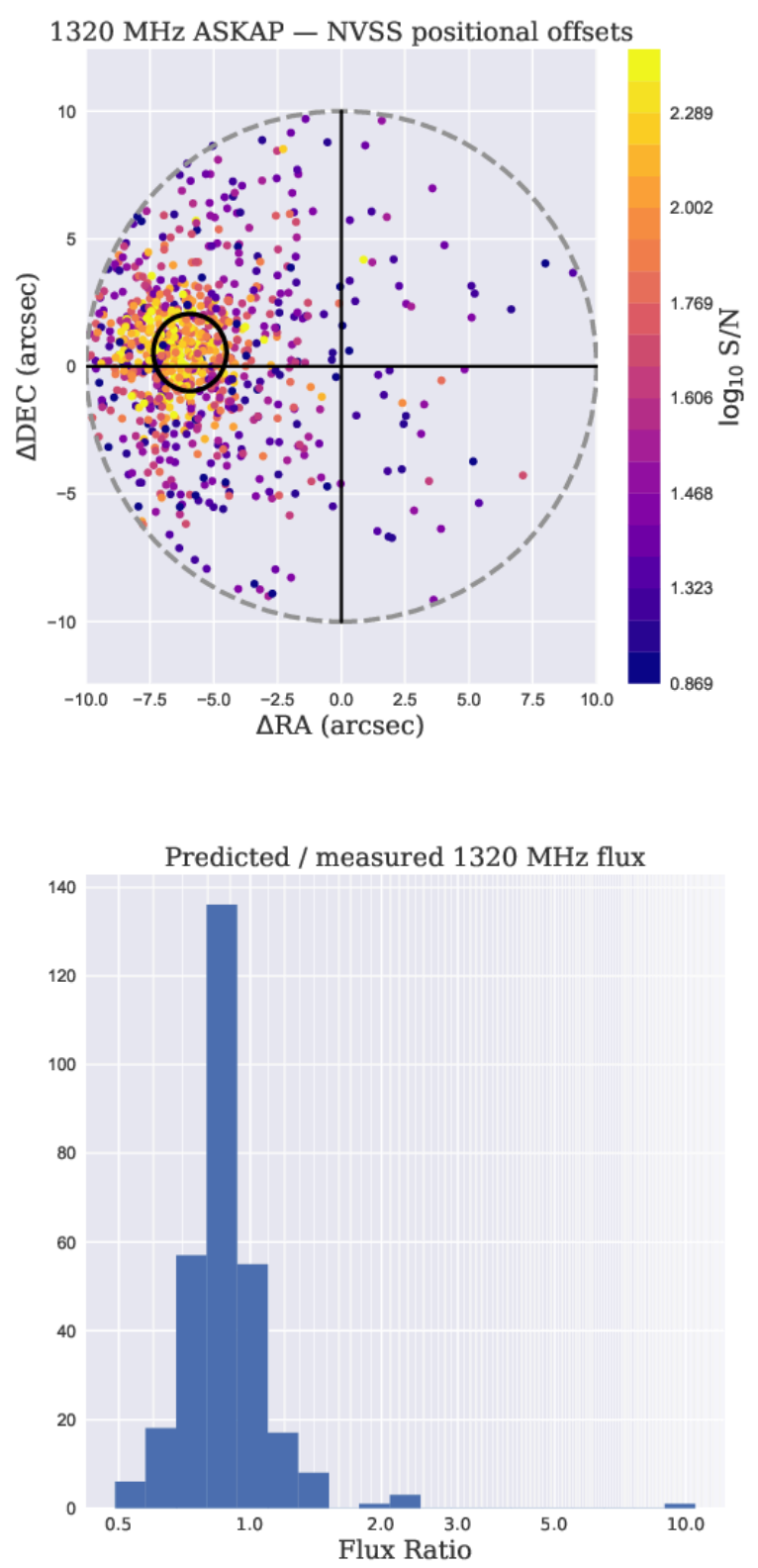

Figure 1. Top panel: offsets of the raw $1320 \mathrm{MHz}$ ASKAP positions from the NVSS positions for sources detected in both surveys. The color bar gives the scale for signal-to-noise (SN) of individual ASKAP sources. The offsets are small compared to the synthesized beam size of $15.8^{\prime \prime}$ by $12.0^{\prime \prime}$ at $1320 \mathrm{MHz}$. The median $1320 \mathrm{MHz}-\mathrm{NVSS}$ offset of $-5.69^{\prime \prime}$ in R.A. and $0.26^{\prime \prime}$ in Dec. was used to correct the raw ASKAP $1320 \mathrm{MHz}$ positions. The median $936 \mathrm{MHz}-\mathrm{NVSS}$ offset of $-6.71^{\prime \prime}$ in R.A. and 1.04" in Dec. was used to correct the raw ASKAP $936 \mathrm{MHz}$ positions. Bottom panel: ratios of NVSS-SUMSS predicted $1320 \mathrm{MHz}$ flux density to ASKAP raw measured $1320 \mathrm{MHz}$ flux density for sources measured in all 3 surveys. The median ratio for $1320 \mathrm{MHz}$ sources of 0.8830 was used to correct the ASKAP $1320 \mathrm{MHz}$ flux densities. The median ratio for $936 \mathrm{MHz}$ sources of 0.8810 was used to correct the ASKAP $936 \mathrm{MHz}$ flux densities. 

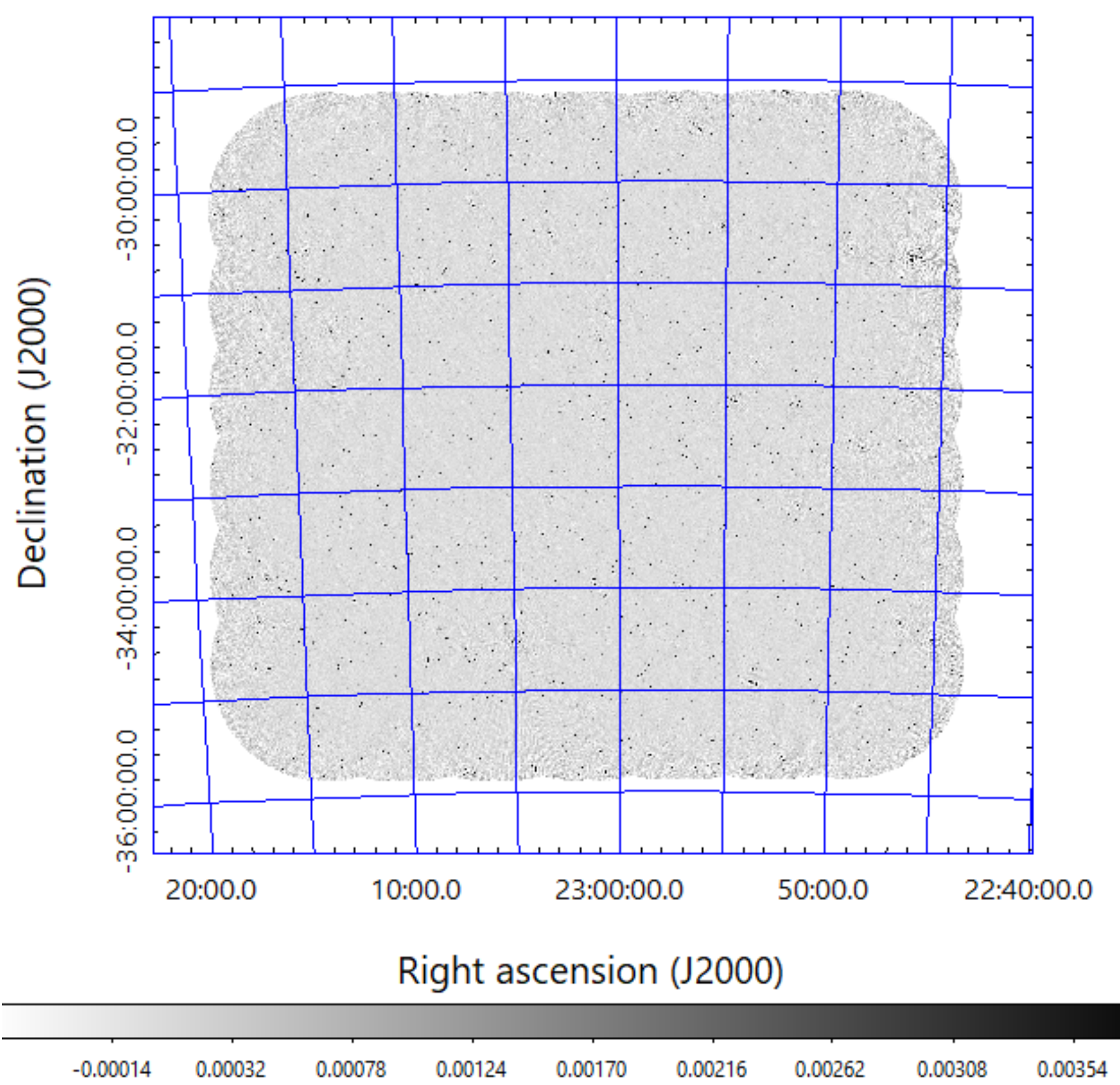

Figure 2. The $936 \mathrm{MHz}$ final processed radio image for the GAMA 23 region. This covers a sky area of $\sim 7.3^{\circ}$ in $\mathrm{R} . \mathrm{A}$. by $\sim 6.8^{\circ}$ in Dec. The greyscale bar at the bottom is in units of Jy/beam. 

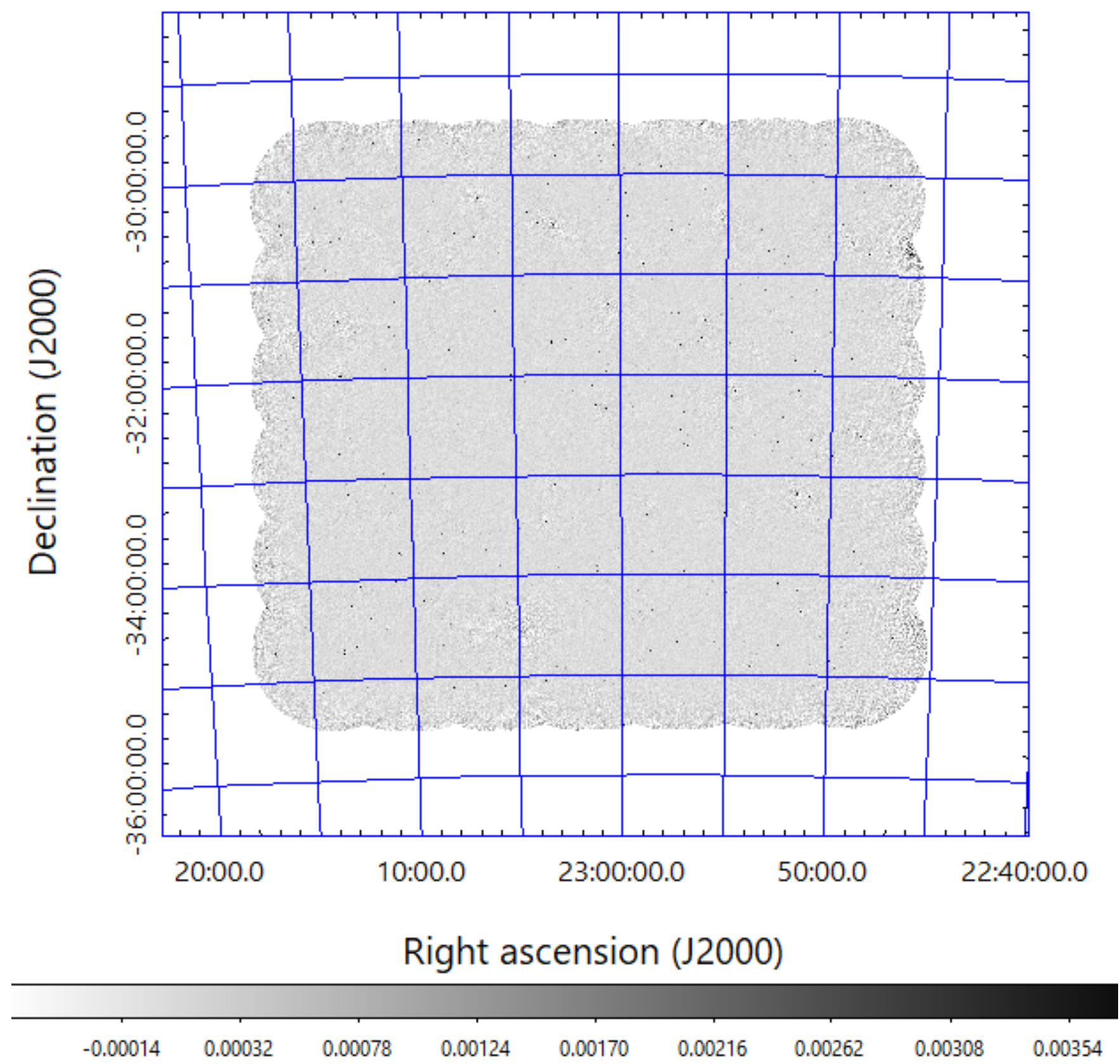

Figure 3. The $1320 \mathrm{MHz}$ final processed radio image for the GAMA 23 region. This covers a sky area of $\sim 6.6^{\circ}$ in $\mathrm{R} . \mathrm{A}$. by $\sim 6.1^{\circ}$ in Dec. The greyscale bar at the bottom is in units of Jy/beam. 
more circular than the beam at lower frequency because of differences in the U-V coverage, which is due primarily to differences in hour angle coverage and different amounts of antenna-based flagging. In both cases, the output images consisted of $7784 \times 7387$ pixel images, where each pixel is a 4 -arcsec square.

Because these data were taken at an early stage of ASKAP commissioning, our flux density and astrometry calibration were still in a preliminary state, and so these had to be corrected after processing. This was done by comparing the ASKAP images with the NVSS (Condon et al., 1998) and SUMSS (Bock et al., 1999) catalogues. We used only non-blended, unresolved sources detected at signal-to-noise (hereafter $\mathrm{SN}$ ) above $5 \sigma$ in ASKAP, NVSS and SUMSS, and which were not flagged as problematic.

The offsets of the raw $1320 \mathrm{MHz}$ ASKAP positions from the NVSS positions are shown in the top panel of Figure 1. The scatter plot for $936 \mathrm{MHz}$ is similar. The medians of the 1320-NVSS and 936-NVSS position offsets were used as the position corrections for the $1320 \mathrm{MHz}$ and $936 \mathrm{MHz}$ images, respectively. The $1 \sigma$ astrometric accuracy was estimated by using the median absolute deviation, converted to $1 \sigma$. At $936 \mathrm{MHz}$ the resulting $1 \sigma$ accuracy is $3.00^{\prime \prime}$ in R.A. and $3.40^{\prime \prime}$ in Dec. and at $1320 \mathrm{MHz}$ the resulting $1 \sigma$ accuracy is $2.37^{\prime \prime}$ in R.A. and 2.64" in Dec.

The flux density scale was calibrated by using sources that were in both NVSS and SUMSS. We interpolated between SUMSS (843 MHz) and NVSS (1420 MHz) flux densities for each source assuming a power-law spectrum. We tabulated the ratios of the calculated $936 \mathrm{MHz}$ and $1320 \mathrm{MHz}$ NVSS-SUMSS flux densities to the raw 936 $\mathrm{MHz}$ and $1320 \mathrm{MHz}$ ASKAP flux densities, respectively. The histogram of these ratios for $1320 \mathrm{MHz}$ is shown in the bottom panel of Figure 1. The median calculated to raw $936 \mathrm{MHz}$ flux density ratio was 0.8810 and the median for $1320 \mathrm{MHz}$ was 0.8830 . These median ratios were used as flux density correction factors for the ASKAP $936 \mathrm{MHz}$ and $1320 \mathrm{MHz}$ images, respectively.

The resulting images are shown in Figures 2 and 3. The root-mean-square (r.m.s.) noise of source free regions in both $936 \mathrm{MHz}$ and $1320 \mathrm{MHz}$ images is $\sim 0.1$ $\mathrm{mJy} /$ beam. To illustrate the image quality obtained with ASKAP in its commissioning phase, we show images on spatial scales of tens of arcminutes. Figure 4 and Figure 5 show regions with typical r.m.s. noise level in the maps $(0.1 \mathrm{mJy})$. The first illustrates some double radio sources. The latter shows one triple source (central source plus two lobes) at the top centre of the image and a fainter triple source at the lower left.

\subsubsection{Source extraction}

We make the distinction between components, which are individual point-like detections in a radio image, and sources, which can be either a single component

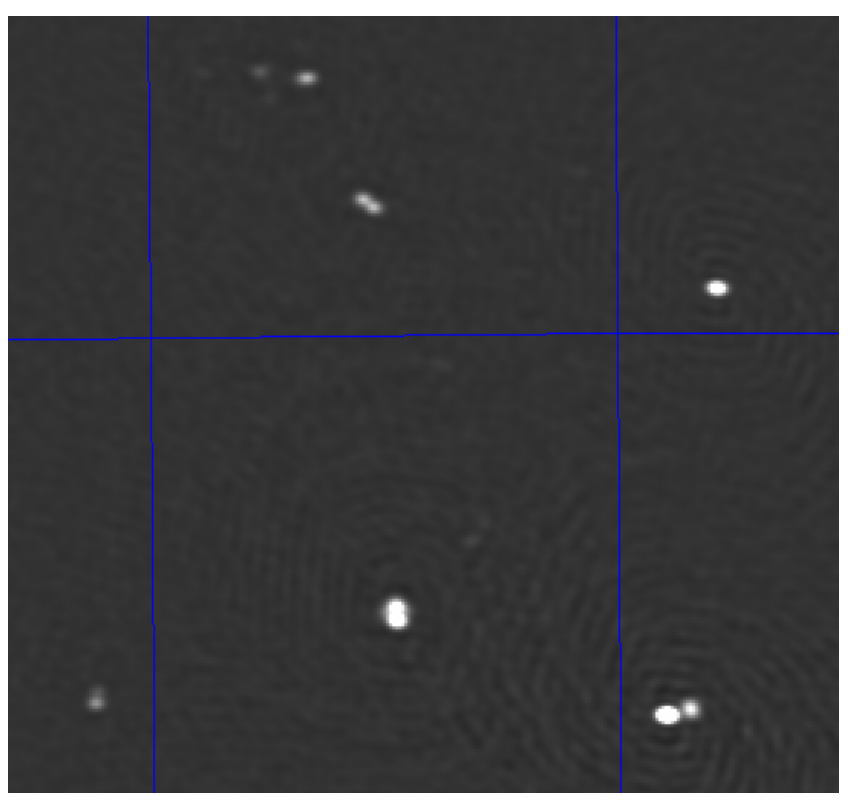

Figure 4. Enlargement of the $1320-\mathrm{MHz}$ image in an area centered on R.A. $23 \mathrm{H} 01 \mathrm{M} 10 \mathrm{~S}$, Dec. $-32^{\circ} 13^{\prime} 30^{\prime}$ in a region containing some double radio sources. The grid spacing is $0.2^{\circ}$. The intensity greyscale is linear, from -5 to $+20 \mathrm{~m} \mathrm{Jy} /$ beam.

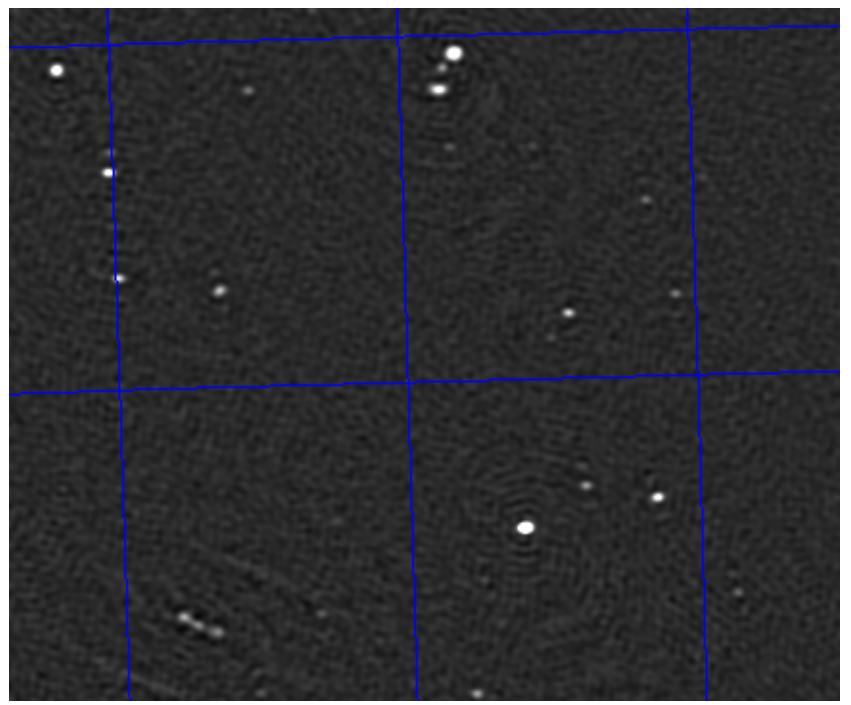

Figure 5. Enlargement of the $1320-\mathrm{MHz}$ image in an area centered on R.A. $23 \mathrm{H} 10 \mathrm{M} 24 \mathrm{~S}$, Dec. $-33^{\circ} 10^{\prime} 30^{\prime}$, in a region containing two radio triples (central source plus two lobes). The grid spacing is $0.2^{\circ}$ in R.A and in Dec. The intensity greyscale is linear, from -1 to $+5 \mathrm{mJy} /$ beam. 
Table 1 ASKAP Observation Details

\begin{tabular}{lllll}
\hline $\begin{array}{l}\text { Scheduling } \\
\text { Block ID }\end{array}$ & $\begin{array}{l}\text { Frequency } \\
(\mathrm{MHz})\end{array}$ & $\begin{array}{l}\text { Bandwidth } \\
(\mathrm{MHz})\end{array}$ & Date & $\begin{array}{l}\text { Observing } \\
\text { time (hrs) }\end{array}$ \\
\hline 2827 & 1320 & 144 & 03-Dec-2016 & 5 \\
2831 & 1320 & 144 & 04-Dec-2016 & 5 \\
2949 & 936 & 192 & 16-Dec-2016 & 4 \\
2955 & 936 & 192 & 17-Dec-2016 & 4 \\
2961 & 936 & 192 & 18-Dec-2016 & 4 \\
\hline
\end{tabular}

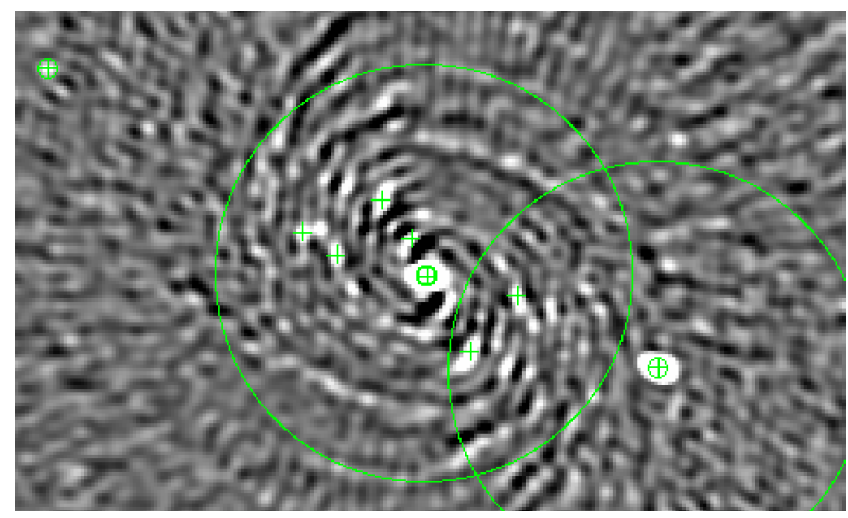

Figure 6. Enlargement of the $936 \mathrm{MHz}$ image centred on a bright component (integrated flux density $0.78 \mathrm{Jy}$ ) which exhibits a spiral shaped pattern of residuals. The large circles are $240^{\prime \prime}$ in radius. The crosses show the detected components (4 sigma above background) before the correction. The small circles show the components after the correction. The intensity greyscale is expanded to show faint features, and is linear from $-1 \mathrm{mJy} / \mathrm{beam}$ (black) to $+1.5 \mathrm{mJy} /$ beam (white). Most sources are saturated at this scale.

or composed of multiple components, such as a doublelobed radio galaxy. The Aegean software (Hancock et al., 2012) was used for component extraction. We used a $4 \sigma$ threshold, resulting in a list of 6053 components at $936 \mathrm{MHz}$, and 3827 at $1320 \mathrm{MHz}$.

Small but significant residuals, of order $1 \%$ of the peak flux density, are found around bright components. The residuals occur in a spiral pattern which extends out to a radius of about $6^{\prime}(\sim 10$ times the synthesized beam). Figure 6 shows a bright component exhibiting such residuals, the resulting false components, and our correction for them. The flux densities of the false components were all less than $1.25 \%$ of the integrated flux density of the bright central component at $936 \mathrm{MHz}$, and less than $1.6 \%$ at $1320 \mathrm{MHz}$. We started with the brightest component in the map and labelled it as the "source". All components within $6{ }^{\prime}$ radius of the "source" which were fainter than $1.25 \%$ (at $936 \mathrm{MHz}$ ) or $1.6 \%$ (at $1320 \mathrm{MHz}$ ) of the "source" were added to a list of possible false components. This was repeated for the second brightest component in the map, and so on, until a complete list of possible false components was created. The false components were removed from the original component list except for those few not consistent with
Table 2 Number of sources, by morphology, in the radio source catalogue

\begin{tabular}{lcccc}
\hline Frequency & total & single & $\begin{array}{c}\text { double } \\
(2 \text { lobes })\end{array}$ & $\begin{array}{c}\text { triple } \\
\text { (core+2 lobes) }\end{array}$ \\
\hline $936 \mathrm{MHz}$ & 5791 & 5710 & 56 & 25 \\
$1320 \mathrm{MHz}$ & 3589 & 3451 & 109 & 29 \\
\hline
\end{tabular}

the spiral beam residual pattern. After artifact removal, the catalogue contained 5968 components at $936 \mathrm{MHz}$, and 3757 at $1320 \mathrm{MHz}$.

\subsubsection{Source counts}

The source counts normalized by the Euclidean slope $\left(S^{2.5} d N / d S\right.$ vs. flux density), are shown in Figure 7 for $936 \mathrm{MHz}$ and $1320 \mathrm{MHz}$. The smooth line is a fit to the compilation of published source count data from Hopkins et al. (2003). The flux densities for $936 \mathrm{MHz}$ sources and $1320 \mathrm{MHz}$ sources were adjusted to $1.4 \mathrm{GHz}$ flux densities using a spectral index of $\alpha=-0.7$.

The data for both frequencies match well the fit line between $\sim 1.2 \mathrm{mJy}$ and $\sim 0.2 \mathrm{Jy}$. Above $0.2 \mathrm{Jy}$, the counts are dominated by small number statistics, reflected in the large error bars. Below $\sim 1.2 \mathrm{mJy}$, the observations fall below the expected source counts, indicating that they are incomplete at this level, particularly at $1320 \mathrm{MHz}$. The map r.m.s. is $0.1 \mathrm{mJy}$ excluding the edges. The r.m.s. rises nearly linearly from to $\sim 0.1 \mathrm{mJy}$ at $\sim 0.5^{\circ}$ from the edges to $\sim 0.5 \mathrm{mJy}$ at the edges. It also rises from $\sim 0.1$ mJy at $\sim 0.25^{\circ}$ away from $\sim 0.5$ Jy sources to $\sim 1 \mathrm{mJy}$ at $\sim 0.05^{\circ}$ away. The fraction of the map area with r.m.s above $\sim 0.1 \mathrm{mJy}$ is $\sim 0.2$. Thus a significant fraction of sources below $\sim 1$ mJy are not detected at the $4 \sigma$ level.

\subsubsection{Identifying complex radio sources}

About $95 \%$ of radio sources at the mJy level, which we term "simple", consist of a single component which is coincident with the optical host galaxy (e.g., Norris et al., 2006). The remaining $\sim 5 \%$ are complex sources, such as double-lobed radio galaxies which appear on the radio image as two isolated components typically separated by tens of arc seconds, with the optical host galaxy located roughly halfway between them. Cross-matching simple sources with optical/IR data is straightforward, and can 


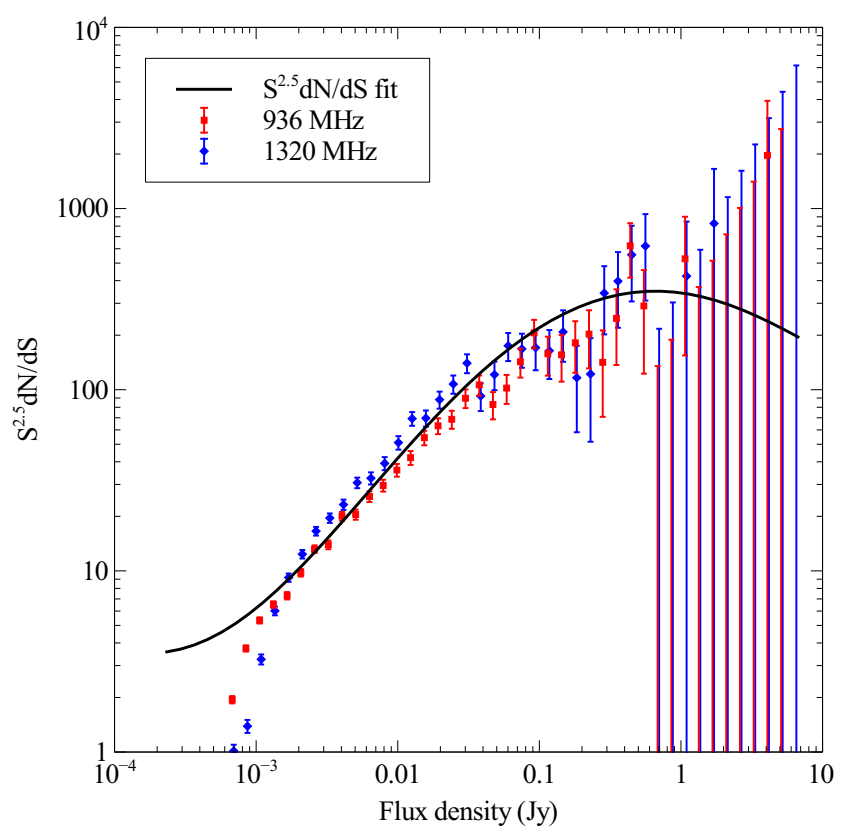

Figure 7. Source counts normalized by the Euclidean slope, $S^{2.5} d N / d S$, from the $936 \mathrm{MHz}$ and $1320 \mathrm{MHz}$ observations, with Poisson error bars. The smooth line is a polynomial fit to the compilation of published source count data (Hopkins et al., 2003).

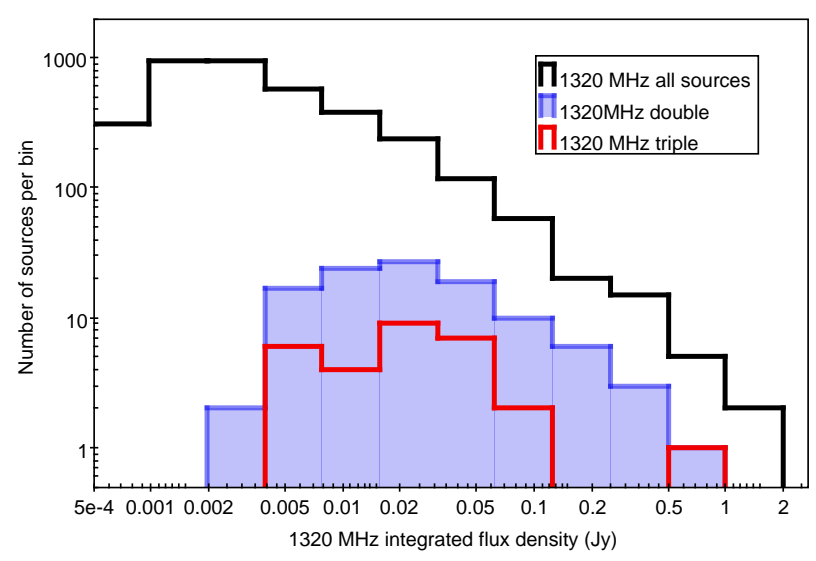

Figure 8. Distribution of integrated $1320 \mathrm{MHz}$ flux densities of all sources (black line), double sources (blue shaded histogram) and triple sources (red line). The all source category is dominated by single sources. The doubles are brighter on average than the triples and both are much brighter than single sources. be achieved using any of several algorithms and tools such as those available within TopCat (Taylor, 2011).

To identify the complex sources, we compiled one list of candidate complex sources from the list of "islands" generated by Aegean, and a second list of candidates by using TopCat to identify pairs and groups of more than two sources within a radius of 6 arcmin. There was a significant overlap $(\sim 50-70 \%)$ between these two lists of candidates. The difference between the two lists illustrates the difficulty of using a single algorithm to identify complex sources. The visual inspection of Aegean islands and TopCat groups resulted in a revised list of singles, doubles and triples. The singles include those components that were put in islands or groups by the software, but had no visual evidence of association. All islands and groups of 4 or more were found to consist of a double or triple plus unrelated singles.

We tested the doubles against the Magliocchetti criterion (Magliocchetti et al., 1998), for which the flux density ratio of the two lobes is required to be between $1 / 4$ and 4 , and the separation is required to be $<100^{\prime \prime}$ $\times \sqrt{S_{\text {tot }} / 100 \mathrm{mJy}}$. We used a flux density ratio between $1 / 3$ and 3 , to err on the side of rejecting sources, but only found one source with flux density ratio between $1 / 4$ and $1 / 3$ (or 3 and 4 ) that also passed the separation test. Sources that failed this test were split into components and added back to the singles list.

We scanned the full ASKAP radio images for radio doubles or triples not identified by either Aegean or TopCat algorithms, and found several more radio doubles and triples, typically those with large angular separation. The resulting numbers of single, double and triple radio sources are shown in Table 2.

To estimate host galaxy positions and integrated flux densities for the double sources, we calculated the flux density-weighted centroid and the total integrated flux density. For the triples, we assumed the host position to be that of the component closest to the centre. The distribution of $1320 \mathrm{MHz}$ integrated flux densities for the double sources, triple source and full set of singles, doubles and triples is show in Fig. 8.

\subsection{Comparison with NVSS and SUMSS surveys}

We compared our $936-\mathrm{MHz}$ and $1320-\mathrm{MHz}$ images and components with the $1420 \mathrm{MHz}$ NVSS and SUMSS $843 \mathrm{MHz}$ catalogues to verify the ASKAP imaging and source detection algorithms.

Figure 9 shows a region from the $936-\mathrm{MHz}$ map comparing the $936-\mathrm{MHz}$ detected components with components from the NVSS and SUMSS catalogues. In the full area covered by the $936-\mathrm{MHz}$ image, there are $5897936-\mathrm{MHz}$ components, 2718 NVSS components and 1152 SUMSS components. There are 2209 936-MHz NVSS cross-matches within $20^{\prime \prime}, 879$ 936-MHz - SUMSS 


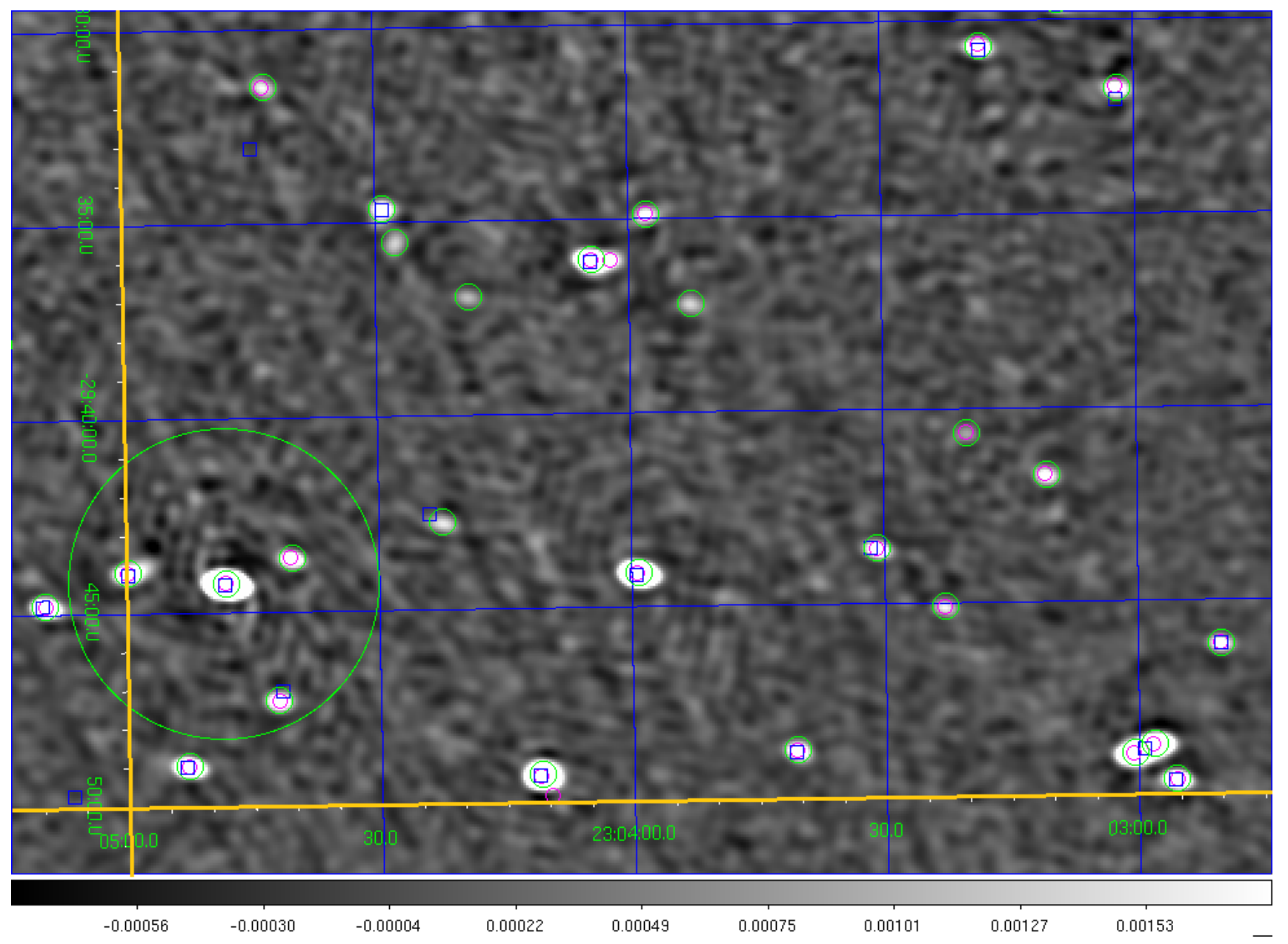

Figure 9. Enlargement of the 936-MHz image in an area centered on R.A. $23 \mathrm{~m} 04 \mathrm{~m}$, Dec. $-29^{\circ} 40^{\prime}$. The small green circles show the 936- $\mathrm{MHz}$ detected sources, the large green circle is the area around a bright source within which false components were removed (see text, Section 2.1.1). Overlaid are the NVSS $1420 \mathrm{MHz}$ sources (small magenta circles) and SUMSS $843 \mathrm{MHz}$ souces (blue squares). The intensity greyscale is linear, from -1 to $+2 \mathrm{mJy} /$ beam, which is expanded to show the background noise level. The grid spacing in Dec. is $5^{\prime}$ and the grid spacing in R.A. is 30 s. 
matches and 943 NVSS - SUMSS matches. In the full area covered by the $1320-\mathrm{MHz}$ image, there are 3756 $1320-\mathrm{MHz}$ components, 1724 NVSS components and 735 SUMSS components. There are 1532 1320-MHz NVSS cross-matches within $20^{\prime \prime}, 702$ 1320-MHz - SUMSS matches and 701 NVSS - SUMSS matches.

All bright components ( $\gtrsim 20 \mathrm{mJy}$ ) are detected in all 3 data sets, ASKAP, SUMSS and NVSS. The number of faint matches is consistent with expectations for detections of real components with noise, given the different sensitivities of the data.

\subsection{Optical Data}

The GAMA G23 imaging and i-band photometric data is from the VST Kilo-Degree Survey (KiDS, de Jong et al. 2013), and ZYJHKs-bands data is from the VISTA Kilo-degree Infrared Galaxy survey (VIKING, Edge et al. 2013). The GAMA photometry is described in Driver et al. (2016) and was obtained from the GAMA Data Management Unit (DMU) "G23InputCatv6". All magnitudes are in the AB system. The G23 spectroscopic sample was VST KiDS i-band selected with a magnitude limit of 19.2. The GAMA spectral catalogue is described in Hopkins et al. (2013). The data reduction, spectral analysis and redshift completeness are described in Liske et al. (2015). The spectral line information is from the GAMA DMU "SpecLineSFRv5". There are 37359 galaxies with spectra and redshifts for the area overlapping the $936 \mathrm{MHz}$ field.

Because full SED-derived stellar mass estimates were not yet available for G23, we obtained stellar mass estimates for G23 galaxies by matching each G23 galaxy to galaxies with similar redshift and iZYJHKs photometry in the GAMA equatorial catalogues, and assuming they had a similar mass. We use a Gaussian weighting kernel, with size of 0.005 in the redshift dimension and sizes of the photometric $1 \sigma$ errors in the photometric dimensions, to find the average mass-to-light ratio of the 'similar' reference galaxies, and assigned that mass to the G23 galaxy. This idea is similar in spirit to kernal-density estimation (KDE) photometric redshift estimation of Wolf et al. (2017). We have validated this approach by testing our ability to recover the SED-fit mass estimates for half of the equatorial catalogue, using the other half of the catalogue as a reference sample. We find that the global random error in the mass estimates derived in this way is of the order of 0.15 dex, compared to a median formal uncertainty of 0.12 dex for the SED-fit mass estimates described in Taylor et al. (2011). This yields 35353 galaxies with mass determinations.

\subsection{IR data}

The IR data were taken from the ALLWISE catalogue of the WISE satellite (Wright et al., 2010). A detailed
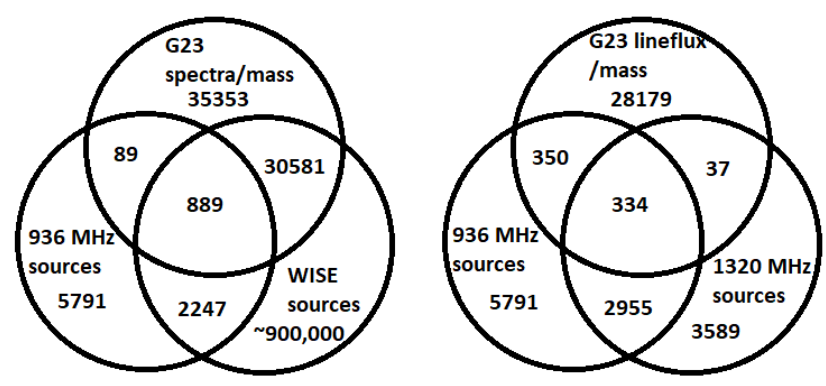

Figure 10. Number of matches between $936 \mathrm{MHz}$ sources, $1320 \mathrm{MHz}$ sources, G23 galaxies and WISE sources. The central set of 889 in the left diagram was obtained by first matching the 35353 G23 galaxies with spectra and masses to the WISE sources, then matching the resulting $30581+889$ galaxies to the $5791936-\mathrm{MHz}$ sources. The central set of 334 in the right diagram was obtained by first matching $5791936-\mathrm{MHz}$ and $35891320-\mathrm{MHz}$ sources, then matching those $2955+334$ to 28179 G23 galaxies with linefluxes and masses.

description of the WISE mission and catalogue is on-line at the Infrared Science Archive (IRSA) at NASA/IPAC (http://irsa.ipac.caltech.edu). The catalogue (hereafter referred to as WISE) contains Vega magnitudes and errors for wavebands at $3.4,4.6,12$, and 22 micron wavelengths (bands W1, W2, W3, W4). The angular resolution is $6.1^{\prime \prime}, 6.4^{\prime \prime}, 6.5^{\prime \prime}$, and $12.0^{\prime \prime}$ in the four bands, respectively. The w1mpro, w2mpro, w3mpro, w4mpro magnitudes and errors were used in the current analysis. The appendix of Cluver et al. (2014) discusses the effect for resolved (nearby) sources on the WISE mpro magnitudes. The w1mpro and w2mpro magnitudes are fainter than isophotal magnitudes by $\sim 0.4$. Here we are using W1-W2 colour which is much less affected for resolved sources.

\section{MULTI-WAVELENGTH ANALYSIS OF THE ASKAP RADIO SOURCES AND G23 GALAXIES}

\subsection{Source cross-matching}

We cross-matched the $936 \mathrm{MHz}$ and $1320 \mathrm{MHz}$ catalogues of radio sources to the G23 galaxies by matching positions within a specified radius using TopCat. For each cross-match, we first plotted the separation distribution for the real catalogues and for the case where one of the catalogues had the positions randomized. Then we compared the separation distributions to determine an optimum radius for separating real from random matches. The $936 \mathrm{MHz}$ and $1320 \mathrm{MHz}$ catalogues were matched using a radius of $7^{\prime \prime}$. The $1320 \mathrm{MHz}$ catalogue was matched with G23 using radius $5^{\prime \prime}$. The $936 \mathrm{MHz}$ catalogue was matched with G23 using radius $7^{\prime \prime}$. Because the $1320 \mathrm{MHz}$ sources were essentially a subset of the $936 \mathrm{MHz}$ sources, we mainly report results for the 
Table 3 Population measures for $936 \mathrm{MHz}$ and $1320 \mathrm{MHz}$ sources, G23 galaxies ${ }^{a}$, and cross-matches with WISE photometry.

\begin{tabular}{lccccc}
\hline Category & No. & $\begin{array}{c}\text { Redshift } \\
\text { mean(SD) }\end{array}$ & $\begin{array}{c}l o g\left(M / M_{\odot}\right) \\
\text { mean(SD) }\end{array}$ & $\begin{array}{c}\text { W1 mag } \\
\text { mean(SD) }\end{array}$ & $\begin{array}{c}936 \mathrm{MHz} \text { f.d.(Jy) } \\
\text { mean(SD) }\end{array}$ \\
\hline $936 \mathrm{MHz}$ & 5791 & $\mathrm{n} / \mathrm{a}$ & $\mathrm{n} / \mathrm{a}$ & $\mathrm{n} / \mathrm{a}$ & $0.0095(0.0413)$ \\
$1320 \mathrm{MHz}$ & 3589 & $\mathrm{n} / \mathrm{a}$ & $\mathrm{n} / \mathrm{a}$ & $\mathrm{n} / \mathrm{a}$ & $\mathrm{n} / \mathrm{a}$ \\
$936 / 1320 \mathrm{MHz}$ & 3289 & $\mathrm{n} / \mathrm{a}$ & $\mathrm{n} / \mathrm{a}$ & $\mathrm{n} / \mathrm{a}$ & $0.0124(0.0506)$ \\
G23 spectra/mass & 35353 & $0.220(0.118)$ & $10.37(0.62)$ & $\mathrm{n} / \mathrm{a}$ & $\mathrm{n} / \mathrm{a}$ \\
G23 mass/lineflux & 28179 & $0.181(0.073)$ & $10.24(0.60)$ & $\mathrm{n} / \mathrm{a}$ & $\mathrm{n} / \mathrm{a}$ \\
G23 mass/4lineflux $>0^{b}$ & 15165 & $0.171(0.075)$ & $9.99(0.60)$ & $\mathrm{n} / \mathrm{a}$ & $\mathrm{n} / \mathrm{a}$ \\
G23 mass/lineflux $<0^{c}$ & 13014 & $0.194(0.069)$ & $10.52(0.46)$ & $\mathrm{n} / \mathrm{a}$ & $\mathrm{n} / \mathrm{a}$ \\
G23 mass/4lineflux $<0^{d}$ & 1785 & $0.186(0.072)$ & $10.61(0.44)$ & $\mathrm{n} / \mathrm{a}$ & $\mathrm{n} / \mathrm{a}$ \\
$936 /$ G23 spectra/mass & 978 & $0.221(0.169)$ & $10.70(0.62)$ & $\mathrm{n} / \mathrm{a}$ & $0.0064(0.0322)$ \\
$936 /$ G23 mass/lineflux & 684 & $0.148(0.083)$ & $10.51(0.61)$ & $\mathrm{n} / \mathrm{a}$ & $0.0043(0.0192)$ \\
936/G23 mass/4lineflux $>0$ & 410 & $0.127(0.075)$ & $10.25(0.59)$ & $\mathrm{n} / \mathrm{a}$ & $0.0037(0.0233)$ \\
$936 /$ G23 mass/lineflux $<0$ & 274 & $0.181(0.084)$ & $10.90(0.41)$ & $\mathrm{n} / \mathrm{a}$ & $0.0051(0.0102)$ \\
936/G23 mass/4lineflux $<0$ & 45 & $0.184(0.068)$ & $10.98(0.39)$ & $\mathrm{n} / \mathrm{a}$ & $0.0096(0.0160)$ \\
G23/WISE spectra/mass & 31470 & $0.223(0.118)$ & $10.41(0.59)$ & $15.39(0.81)$ & $\mathrm{n} / \mathrm{a}$ \\
G23/WISE mass/lineflux & 25234 & $0.184(0.072)$ & $10.28(0.57)$ & $15.42(0.84)$ & $\mathrm{n} / \mathrm{a}$ \\
G23/WISE mass/4lineflux $>0$ & 13149 & $0.174(0.074)$ & $10.05(0.57)$ & $15.69(0.80)$ & $\mathrm{n} / \mathrm{a}$ \\
G23/WISE mass/lineflux $<0$ & 11985 & $0.194(0.068)$ & $10.54(0.45)$ & $15.13(0.79)$ & $\mathrm{n} / \mathrm{a}$ \\
G23/WISE mass/4lineflux $<0$ & 837 & $0.186(0.075)$ & $10.64(0.43)$ & $14.85(0.93)$ & $\mathrm{n} / \mathrm{a}$ \\
936/G23/WISE spectra/mass & 889 & $0.228(0.170)$ & $10.74(0.59)$ & $14.24(0.88)$ & $0.0067(0.0336)$ \\
936/G23/WISE mass/lineflux & 610 & $0.152(0.082)$ & $10.56(0.59)$ & $14.01(0.92)$ & $0.0043(0.0199)$ \\
936/G23/WISE mass/4lineflux $>0$ & 352 & $0.133(0.075)$ & $10.30(0.57)$ & $14.04(0.88)$ & $0.0036(0.0246)$ \\
936/G23/WISE mass/lineflux $<0$ & 258 & $0.179(0.084)$ & $10.92(0.40)$ & $13.97(0.98)$ & $0.0053(0.0104)$ \\
936/G23/WISE mass/4lineflux $<0$ & 23 & $0.177(0.078)$ & $11.03(0.37)$ & $13.89(0.94)$ & $0.0130(0.0181)$ \\
\hline
\end{tabular}

a. The GAMA23 stellar masses were based on stellar masses from the GAMA equatorial fields as described in Section 2.3 .

b. 4lineflux $>0$ means $\mathrm{H} \alpha, \mathrm{H} \beta$, [OIII] $\lambda 5007$ and [SII] $\lambda \lambda 6717,6731$ all have lineflux $>0$.

c. lineflux $<0$ means any one of $\mathrm{H} \alpha, \mathrm{H} \beta$, [OIII] $\lambda 5007$ and [SII] $\lambda \lambda 6717,6731$ has lineflux $<0$.

d. 4lineflux $<0$ means $\mathrm{H} \alpha, \mathrm{H} \beta,[\mathrm{OIII}] \lambda 5007$ and $[\mathrm{SII}] \lambda \lambda 6717,6731$ all have lineflux $<0$. 
larger set of $936 \mathrm{MHz}$ cross-matches.

We cross-matched the $936 \mathrm{MHz}$ and $1320 \mathrm{MHz}$ catalogues and the matched $936 \mathrm{MHz} / 1320 \mathrm{MHz}$ catalogue with WISE using a radius of $4^{\prime \prime}$. This is smaller than for the cross-match of radio catalogues with G23 because of the much higher source density on the sky for WISE than G23.

G23 was cross-matched with WISE using radius of $1.5^{\prime \prime}$. This gives low contamination $(0.84 \%)$, and yields a large fraction of cross-matches $(87.6 \%)$. For the GAMA G12 and G15 fields, Cluver et al. (2014) found similar results: $77 \%$ (for $\mathrm{G} 12$ ) and $88 \%$ (for G15) of the galaxies had WISE W1 and W2 detections at $>5 \sigma$.

We compared the cross-match of the $936 \mathrm{MHz} / \mathrm{WISE}$ catalogue to G23 with the cross-match of the $936 \mathrm{MHz}$ catalogue with the G23/WISE catalogue. The latter yields better results: the number cross-matches is somewhat reduced, but there is much lower contamination with random cross-matches. The reason for this is the superior spatial resolution of both G23 positions (subarcsec) and WISE positions $\left(\simeq 0.2-0.3^{\prime \prime}\right)$ compared to the uncertainties of the radio positions $\left(\sim 5^{\prime \prime}\right) .^{1}$

From these different catalogues, we then extracted the following subsets:

1. all galaxies with spectra and masses;

2. all galaxies with spectra, masses and BPT line flux measurements;

3. all galaxies with spectra, masses and all BPT lines in emission;

4. all galaxies with spectra, masses and at least one BPT line in absorption;

5. all galaxies with spectra, masses and all BPT lines in absorption.

Summaries of the numbers of sources in the different subsets are given in Table 3 and, in part, in Figure 10. All galaxies with spectra have redshifts.

\subsection{Properties of cross-matched sources}

A mass-redshift diagram for G23 galaxies and their $936 \mathrm{MHz}$ radio counterparts is given in the top panel of Figure 11. At all redshifts, the radio sources occur in the high-mass end of the galaxy mass distribution. The radio source mean masses ('936/G23 spectra/mass' and '936/G23/WISE spectra/mass') are significantly higher than for the full galaxy population ('G23 spectra/mass'), by factors of 2.14 and 2.34 , respectively. The $936 \mathrm{MHz}$ and $1320 \mathrm{MHz}$ mass distributions are not significantly different.

1 The WISE catalogue contains sources detected at $\geq 5 \sigma$, thus does not contain all galaxies with emission in the WISE bands. Forced photometry at the positions of the G23 galaxies would likely have found many $<5 \sigma$ WISE sources associated with the galaxies, although that is beyond the scope of the current work.
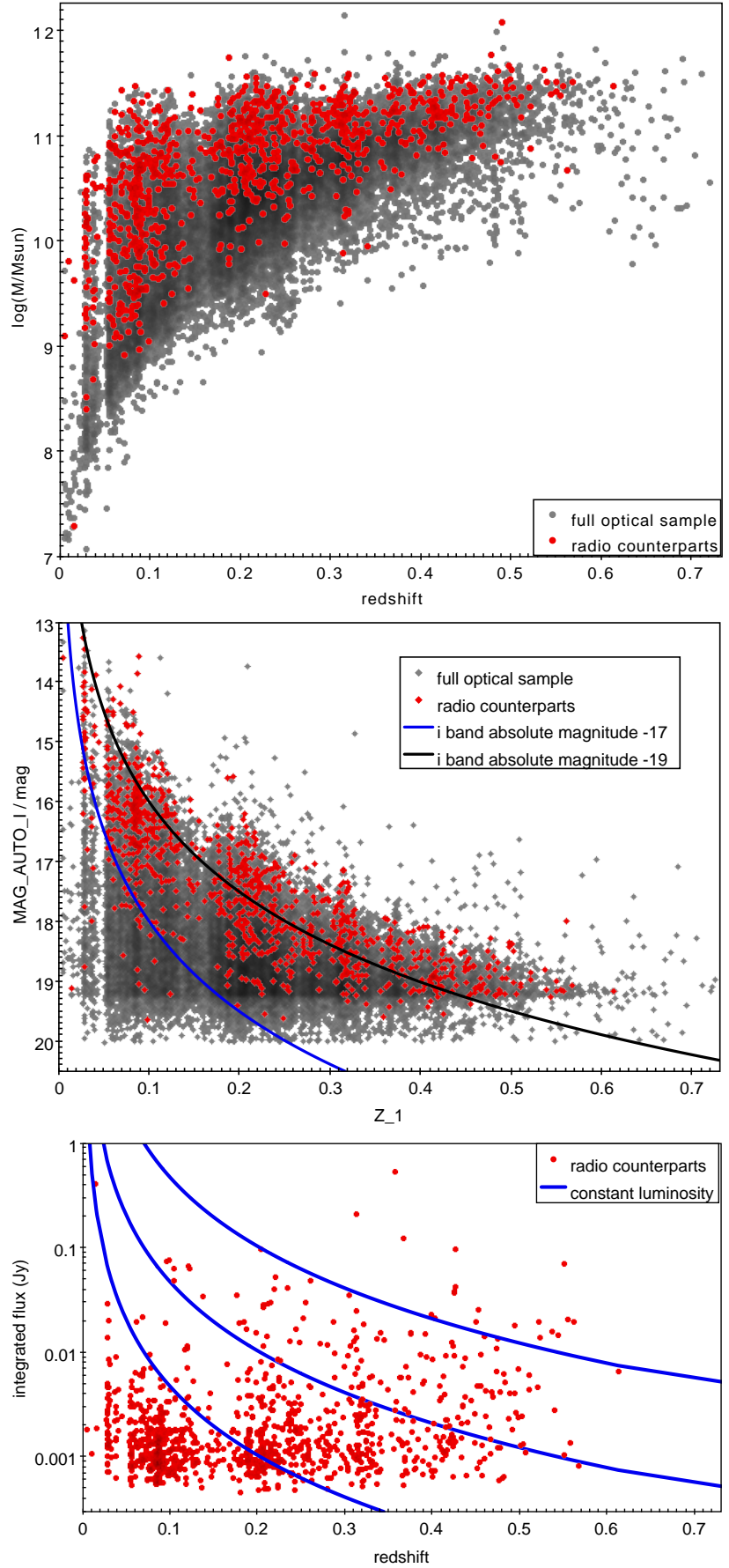

Figure 11. Top panel: Stellar mass in units of $\log \left(M / M_{\odot}\right)$ vs redshift for the $936 \mathrm{MHz} / \mathrm{G} 23$ sources (red), and for all G23 galaxies (grey). This shows that galaxies with detected radio emission are mainly galaxies with larger masses, and shows the effect of redshift-dependent completeness on the lower limit of detected G23 galaxies and radio counterparts. Middle panel: i-band magnitude vs redshift for the galaxy sample and for the galaxies with detected $936 \mathrm{MHz}$ radio sources. The lines are for a galaxy of absolute i band magnitudes -17 and -19 . Bottom panel: Integrated $936 \mathrm{MHz}$ flux density vs redshift for the G23 galaxies with radio counterparts (red points). The blue lines are for sources of constant radio luminosity (differing by one order of magnitude). Radio counterparts which would have flux densities below the limits of the current ASKAP data fall below a horizontal line at $\sim 5 \times 10^{-4} \mathrm{Jy}$. Radio counterparts which would be beyond the current redshift limit of the G23 galaxies fall to the right of a line at $z \sim 0.6$. 
The middle panel of Figure 11 shows i-band magnitude vs redshift for the G23 galaxies and for radio counterparts compared to lines of constant i-band absolute magnitude -17 and -19 . The radio sources are associated with the optically most luminous galaxies, consistent with them having higher than average stellar masses. The fact that the radio counterparts extend down to the detection limit of the G23 region (a horizontal line at $i$ band magnitude of 19.2) implies that the radio observations are fairly complete for redshifts $z<0.6$.

The bottom panel of Figure 11 shows integrated radio flux density vs redshift for the $936 \mathrm{MHz}$ radio counterparts. Lines of constant radio luminosity are shown. Cosmological volume increases rapidly with redshift so the number of luminous radio sources (e.g. within a band between a pair of blue lines) should increase rapidly. This illustrates increasing incompleteness with redshift of radio counterparts and of galaxies.

There are two reasons why we do not observe more radio counterparts:

- Radio counterparts are not detected below the lower edge of the observed distribution because of the limited sensitivity of the radio observations.

- Radio counterparts are not observed at high redshift $(z>0.6)$ because of the lack of such galaxies in G23. The gradual decrease in radio counterparts between redshift 0.1 and 0.6 is consistent with the decrease in the number (i.e. completeness) of optically measured galaxies with redshift, as seen in the top and middle panels.

Both factors contribute to lack of observed radio counterparts. The number of observed radio sources (5791 at $936 \mathrm{MHz}$ ) is much larger than the number with optical counterparts (978). Those without optical galaxy counterparts may lie either at $z<0.6$ with their optical counterpart fainter than the G23 magnitude limit, or at $z>0.6$. The relatively high radio flux density limit of these observations suggests that most are likely to be high radio luminosity AGNs at high redshifts (e.g., Seymour et al., 2008) or, possibly, dust obscured galaxies at lower redshifts $(z \lesssim 0.6)$.

Radio sources, such as broad-line AGN and star forming galaxies, typically have bluer near-ultraviolet (NUV) $-r$ colours than the overall galaxy population (Heckman \& Best, 2014). We used NUV magnitudes from the G23 input catalogue derived from GALEX observations (Liske et al., 2015). The mean NUV magnitude is 21.11 for galaxies detected at $936 \mathrm{MHz}$ compared to 21.94 for all galaxies. For NUV magnitudes brighter than 19, the probability of a galaxy to have radio emission is higher by a factor of $\sim 5$. For SFG, brighter UV magnitudes imply higher star formation rates (Brown et al., 2017). The UV could be from accretion disks of AGN, however recent measurements of the
AGN UV luminosity function (Ricci et al., 2017) ${ }^{2}$ imply that the UV input to the universe from SFG is significantly larger than from AGN. Thus the brighter UV from radio-detected galaxies in most cases is dominated by star formation.

The brightest emission line galaxies in either $\mathrm{H} \alpha$ or $\mathrm{H} \beta$ are mostly detected as $936 \mathrm{MHz}$ sources: 8 out of the 10 brightest $\mathrm{H} \alpha$ emitters and 7 out of the 10 brightest $\mathrm{H} \beta$ emitters are $936 \mathrm{MHz}$ sources whereas only 1 in 25 galaxies with $\mathrm{H} \alpha$ and $\mathrm{H} \beta$ emission lines is a $936 \mathrm{MHz}$ source. Thus, radio sources are highly over-represented in the population of brightest $\mathrm{H} \alpha$ and $\mathrm{H} \beta$ galaxies.

Compared to the full optical sample, the fraction of strong $\mathrm{H} \beta$ absorption line systems is larger by a factor of $\simeq 2$ for the radio counterparts. The mass distributions are shown in the top panel of Figure 12. The mean $\log \left(\mathrm{M} / M_{\odot}\right)$ is 10.37 for the whole galaxy sample, 10.68 for the optical galaxy $\mathrm{H} \beta$-absorption-line sample, and 11.00 for the $936 \mathrm{MHz}$ detected $\mathrm{H} \beta$-absorption-line sample. The stronger $\mathrm{H} \beta$ absorption line strengths for $936 \mathrm{MHz}$ galaxies are related to the higher masses for $936 \mathrm{MHz} \mathrm{H} \beta$ absorption line systems. This is similar to the result from Best et al. (2005) that the fraction of galaxies that host a radio AGN rises strongly with host stellar mass. While the radio flux density limit of the current data is significantly lower than in that work, and the current set of radio sources includes a larger fraction of SFG, it is clear that the highest mass systems include those with old stellar populations, and their radio emission is likely driven by an AGN.

Radio sources have much stronger mid-IR emission than non-radio galaxies in general (see Table 3 ). The 936/G23/WISE sources have a mass about a factor of $\sim 4$ higher than the G23/WISE galaxy population. We created a sample of not936/G23/WISE galaxies by excluding the radio-emitters. Then we selected a massmatched set of non-radio galaxies by choosing the best match by mass from the not936/G23/WISE set for each radio galaxy in the $936 / \mathrm{G} 23 / \mathrm{WISE}$ set. We verified that the mass distribution of the not936/G23/WISE/massmatched set is essentially identical to that of the 936/G23/WISE set. The resulting $22 \mu \mathrm{m}$ luminosity distributions of the radio and non-radio emitters is shown in the bottom panel of Figure 12. There is no difference, except for the 3 radio sources with highest $22 \mu \mathrm{m}$ luminosity, so we can conclude that the higher midIR emission of radio sources is consistent with being associated with increased mass of the galaxy.

Both $22 \mu \mathrm{m}$ and $\sim 1.4 \mathrm{GHz}$ luminosities are star formation rate indicators for SFG (e.g. Kennicutt et al. 2009, Rieke et al. 2009, Brown et al. 2017, Cluver et al. 2017). For AGN, they are indicators of AGN activity (Gürkan et al., 2014). We show the tight correlation between

\footnotetext{
${ }^{2}$ Ricci et al. (2017) also noted that the SF in AGN contributed a significant fraction of the total UV emitted by AGN.
} 

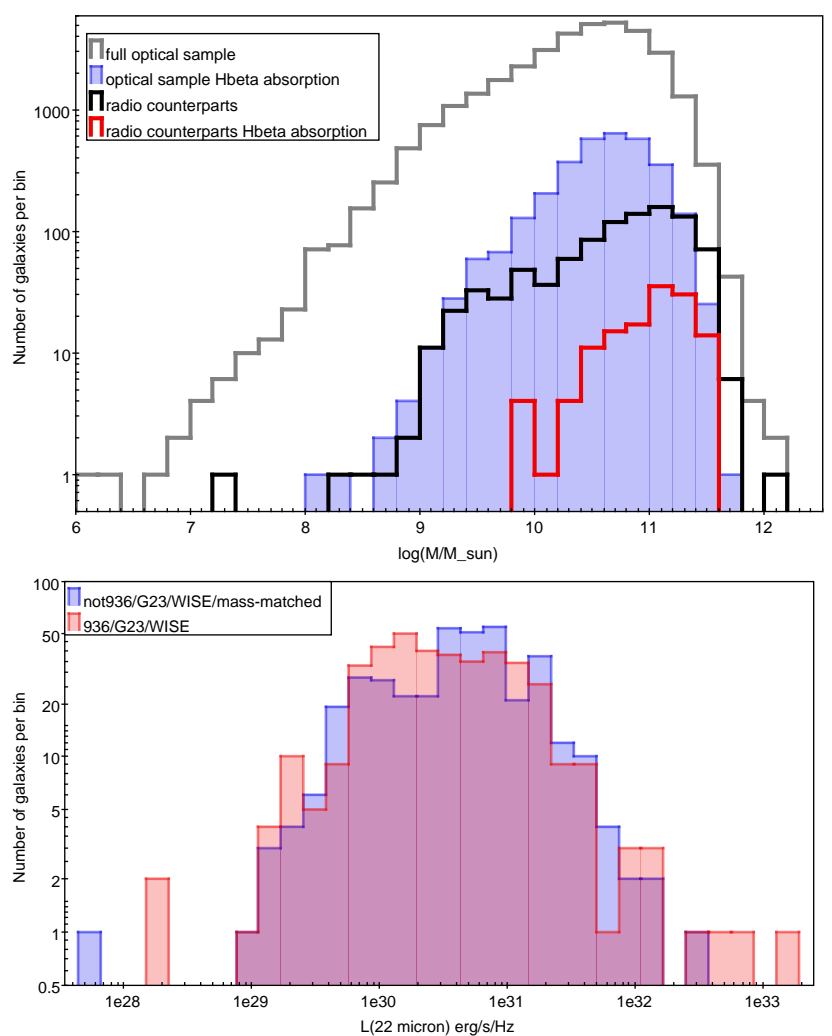

Figure 12. Top panel: The distribution of stellar masses (number of galaxies per mass bin, units of $\left.\log \left(M / M_{\odot}\right)\right)$ for radio counterparts $(936 / \mathrm{G} 23$ set) with $H \beta$ in absorption (red), and for all radio counterparts (black). Overlaid on these are the distributions for G23 galaxies with $H \beta$ in absorption (blue), and for all G23 galaxies (grey). This illustrates (i) the significantly larger masses for galaxies with $H \beta$ in absorption compared to all galaxies; and (ii) the shift to even larger masses for both sets of galaxies when they have $936 \mathrm{MHz}$ radio emission. Bottom panel: comparison of the $22 \mu \mathrm{m}$ luminosity distribution for radio-emitting galaxies (red histogram) with that for a mass-matched set of non-radio galaxies (blue histogram).
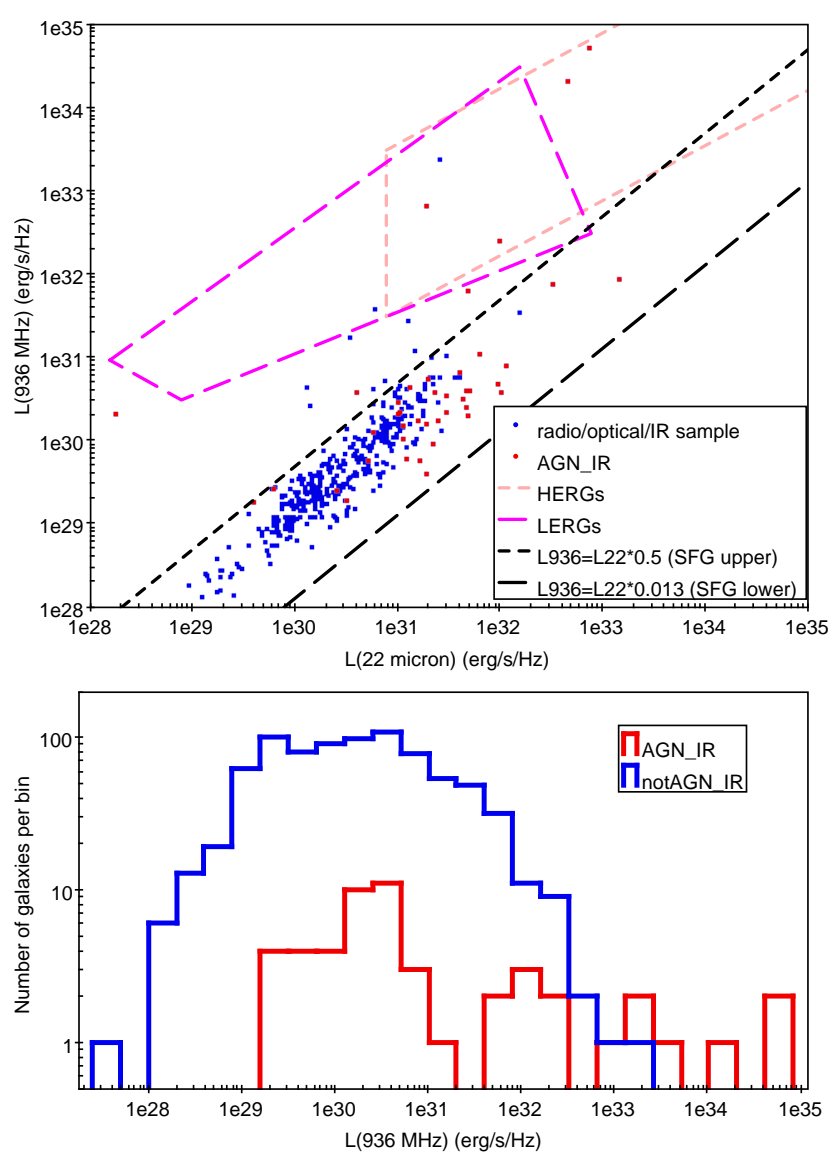

Figure 13. Top panel: $936 \mathrm{MHz}$ luminosity vs W4 band $(22 \mu \mathrm{m})$ luminosity, both in units of $\mathrm{erg} \mathrm{s}^{-1} \mathrm{~Hz}^{-1}$, for the 936/G23/WISE sources with WISE W4 signal-to-noise (SN) $\geq 3$ (blue points) and for the $\mathrm{AGN}_{I R}$ subset (red points). Most sources $(96 \%, 436$ of 452) fall in the band for star-forming galaxies, delimited by the two lines shown (derived from Rieke et al. 2009). The regions outlined by the pink and magenta dashed lines indicate the regions occupied by HERGs and LERGs (Gürkan et al., 2014). The sources detected here mainly have lower $L_{936 ~} \mathrm{M} \mathrm{Hz}_{\mathrm{z}}$ than HERGs and LERGs. Bottom panel: $936 \mathrm{MHz}$ luminosity distribution for $\mathrm{AGN}_{I R}$ and notAGN ${ }_{I R}$ from the $936 / \mathrm{G} 23 /$ WISE sample. 
$22 \mu \mathrm{m}$ and $936 \mathrm{MHz}$ luminosities $\left(L_{\nu}\right.$, units erg/s/Hz) for the 936/G23/WISE galaxies in the top panel of Figure $13 .{ }^{3}$ We also show the range of ratio $936 \mathrm{MHz}$ to $22 \mu \mathrm{m}$ luminosity found by Rieke et al. (2009) for SFG by the two black dashed lines. $68 \%(95 \%)$ of the data falls within $\pm 0.3 \mathrm{dex}( \pm 0.6 \mathrm{dex})$ of a $936 \mathrm{MHz}$ to $22 \mu \mathrm{m}$ luminosity ratio of 0.13 . Consistent with our results, Brown et al. (2017) finds that $150 \mathrm{MHz}, 1.4 \mathrm{GHz}$ and $22 \mu \mathrm{m}$ luminosities as a function of $\mathrm{H} \alpha$ luminosity have scatter of 0.2 dex. Thus it is seen that the majority of our radio/optical/IR galaxies are consistent with their radio and $22 \mu \mathrm{m}$ emission powered by star formation.

\subsection{AGN and SFG diagnostics}

\subsubsection{IR AGN diagnostic}

The WISE mid-IR colour criterion W1-W2 $>0.8$ was introduced by Jarrett et al. (2011) to distinguish AGN from other galaxies. Stern et al. (2012) found that AGN can be identified, with $95 \%$ reliability and $78 \%$ completeness, using this criterion. Sources with W1-W2 $>0.8$ $(>1 \sigma)$ are labelled $\mathrm{AGN}_{I R}$ and those with W1-W2<0.8 $(>1 \sigma)$ are labelled notAGN ${ }_{I R}$. The W1-W2 test gives an $\mathrm{AGN}_{I R}$ classification for 374 sources and a notAGN $I R$ classification for 29983 sources.

The $\mathrm{AGN}_{I R}$ and notAGN $I R$ galaxies have strikingly different properties. Figure 13 (top panel) shows the $\mathrm{AGN}_{I R}$ constitute $\sim 1 / 2$ of the outliers from the radio vs. $22 \mu \mathrm{m}$ relation, whereas they are only a small fraction (0.08) of the radio and $22 \mu \mathrm{m}$ emitting galaxies. The bottom panel of Figure 13 shows the radio luminosity distributions of $\mathrm{AGN}_{I R}$ and notAGN $I R$. The $\mathrm{AGN}_{I R}$ are more luminous on average by a factor of 156 at $936 \mathrm{MHz}$.

Figure 14 shows the redshift, mass and $22 \mu \mathrm{m}$ luminosity distributions for G23/WISE and 936/G23/WISE samples. The mean redshift of $\mathrm{AGN}_{I R}$ is much higher than for notAGN $I R$ (see Table 4). The mean mass of G23/WISE AGN IR is nearly the same as for notAGN $I R$, and the mass distributions are nearly the same (Figure 14 middle panel). The mean $22 \mu \mathrm{m}$ luminosity of $\mathrm{AGN}_{I R}$ is higher by a factor $\sim 25$ than for notAGN $I R$ (for the G23/WISE w4snr $>3$ sample).

For the $936 \mathrm{MHz}$ detected G23/WISE galaxies (whole and $\mathrm{w} 4 \mathrm{snr}>3$ subsample, see Table 4 ), the $\mathrm{AGN}_{I R}$ redshifts and $936 \mathrm{MHz}$ radio flux densities are higher (by factor $\sim 3$ for redshift and $\sim 8$ for flux density) than for notAGN ${ }_{I R}$. There is an even more extreme difference considering luminosities: the $22 \mu \mathrm{m}$ luminosities are higher by a factor of 20 than for notAGN $I R$ (for

\footnotetext{
${ }^{3}$ The conversion from W4 magnitude to flux density in Jy is given in the WISE explanatory supplement on the NASA/IPAC/IRSA website. The effective wavelength of the W4 filter has been recalibrated to $23 \mu \mathrm{m}$ (Brown et al., 2014a). No K-corrections were applied to obtain $22 \mu \mathrm{m}$ luminosities in this study.
}

the $\mathrm{w} 4 \mathrm{snr}>3$ subsample). The $\mathrm{AGN}_{I R}$ mean masses are similar for the whole 936/G23/WISE sample and the subsample with $\mathrm{w} 4 \mathrm{snr}>3$. However the notAGN $I R$ mean mass is higher (by a factor of 2.0) for the whole 936/G23/WISE sample compared to the subsample with $\mathrm{w} 4 \mathrm{snr}>3$.

The fact that such strong differences in redshift and luminosity are seen between the $\mathrm{AGN}_{I R}$ and notAGN $I R$ categories indicates that the contamination of $\mathrm{AGN}_{I R}$ by notAGN galaxies and of notAGN ${ }_{I R}$ by AGN is not a large fraction of either category. Contamination of not $\mathrm{AGN}_{I R}$ by low luminosity AGN would not strongly affect the redshift and radio luminosity difference we see between the $\mathrm{AGN}_{I R}$ and notAGN $I R$ sets.

\subsubsection{Optical AGN/SFG diagnostic}

As a complement to the W1-W2 colour criterion, we use the BPT diagnostic to distinguish AGN from SFG. Requiring observed BPT line fluxes rejects all galaxies which have $z>0.315$ because the [SII] $\lambda \lambda 6717,6731$ lines are redshifted out of the observed spectra. This has the side effect of rejecting high mass galaxies $(\sim 1 / 3$ to $2 / 3$ of those above $5 \times 10^{10} M_{\odot}$ ).

To classify a galaxy as $\mathrm{AGN}_{\text {opt }}$ or $\mathrm{SFG}_{o p t}$, we required the entire error box in the BPT diagram to lie on the $\mathrm{AGN}_{\text {opt }}$ or $\mathrm{SFG}_{\text {opt }}$ side of the diagnostic BPT line. Figure 15 shows the BPT diagram. Galaxies which have an ambiguous classification because of line flux uncertainties are shown by the grey points.

For the G23 sample, about half (Table 3) of the galaxies with measured line fluxes have one or more of the BPT lines in absorption, and of the $\sim 15,000$ with emission lines, $\sim 4400$ had high enough $\mathrm{SN}$ to be classified by the BPT diagnostic. Table 4 gives the numbers of AGN and SFG for different subsets.

The mass distributions for the different categories are shown in the top and bottom panels of Figure 16. In order, from highest mean mass to lowest mean mass, the categories are: galaxies with all BPT lines in absorption; galaxies with at least one BPT line in absorption; $\mathrm{SFG}_{\text {opt }}$; $\mathrm{AGN}_{\text {opt }}$; and galaxies with all BPT lines in emission. The $\mathrm{AGN}_{\text {opt }}$-classified galaxies have the broadest mass distribution, inconsistent with single-peaked distribution and characteristic of a bimodal mass distribution. A $\chi^{2}$ fit to the distribution shows it requires two components: a high mass component peaking at $\sim 3 \times 10^{10} M_{\odot}$, and a low mass component peaking at $\sim 2 \times 10^{9} M_{\odot}$.

To compare the BPT criterion with the W1-W2 criterion, we use the G23/WISE sample. The requirement of a WISE detection in effect rejects low mass galaxies (see Table 3): it results in rejection of $\sim 1 / 6$ or more of the galaxies below $3 \times 10^{9} M_{\odot}$. Table 4 shows that the increase in mean mass for G23/WISE compared to G23 depends on the AGN classification: G23/WISE AGN has mean mass higher than G23 AGN opt by factor 2.24;

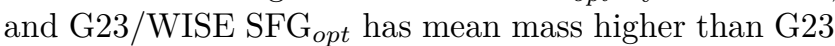


Table 4 Properties ${ }^{a}$ of SFG and AGN subsets using BPT and W1-W2 criteria.

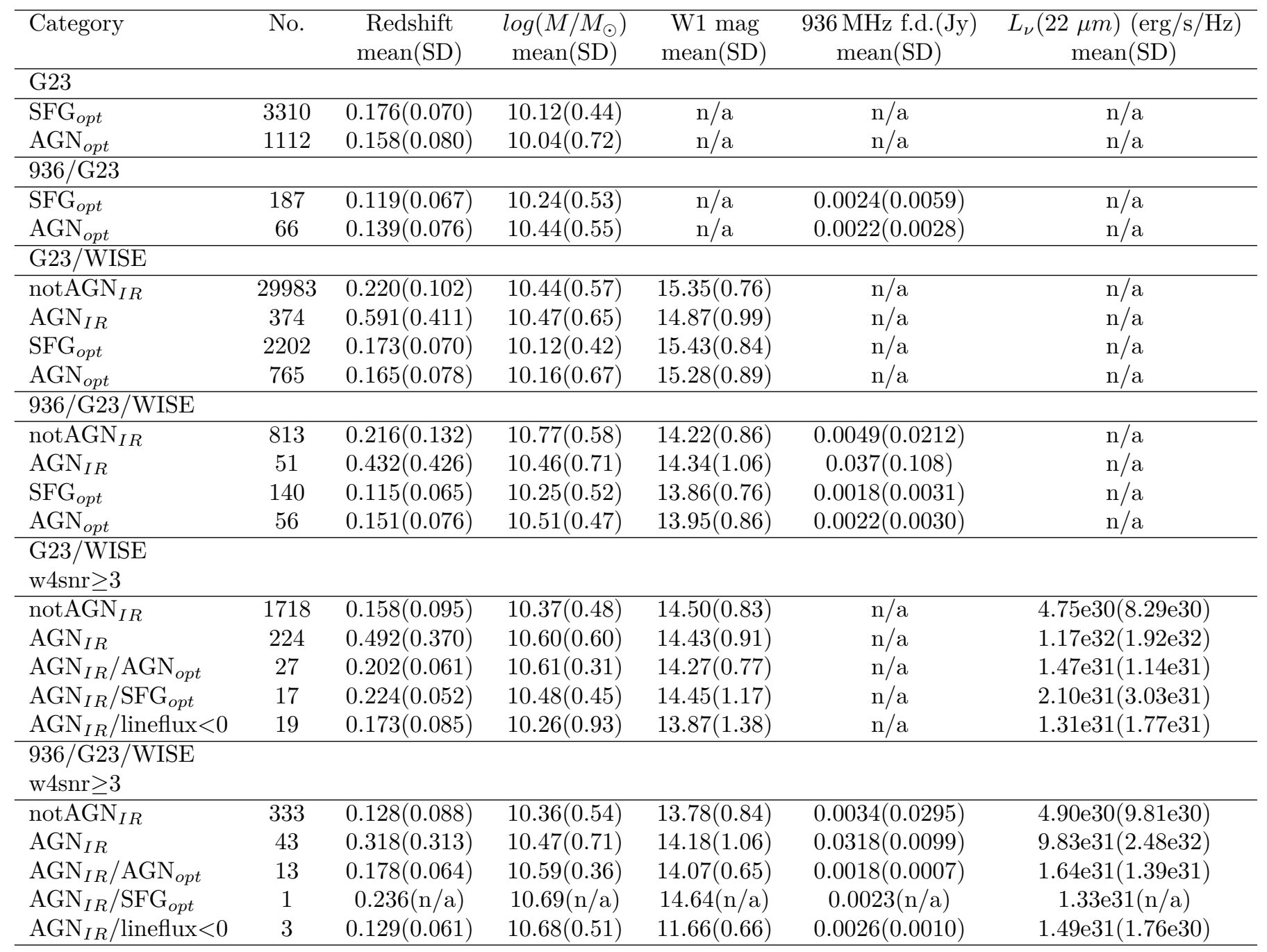

a. The GAMA23 stellar masses were based on stellar masses from the GAMA equatorial fields as described in Section 2.3 . 

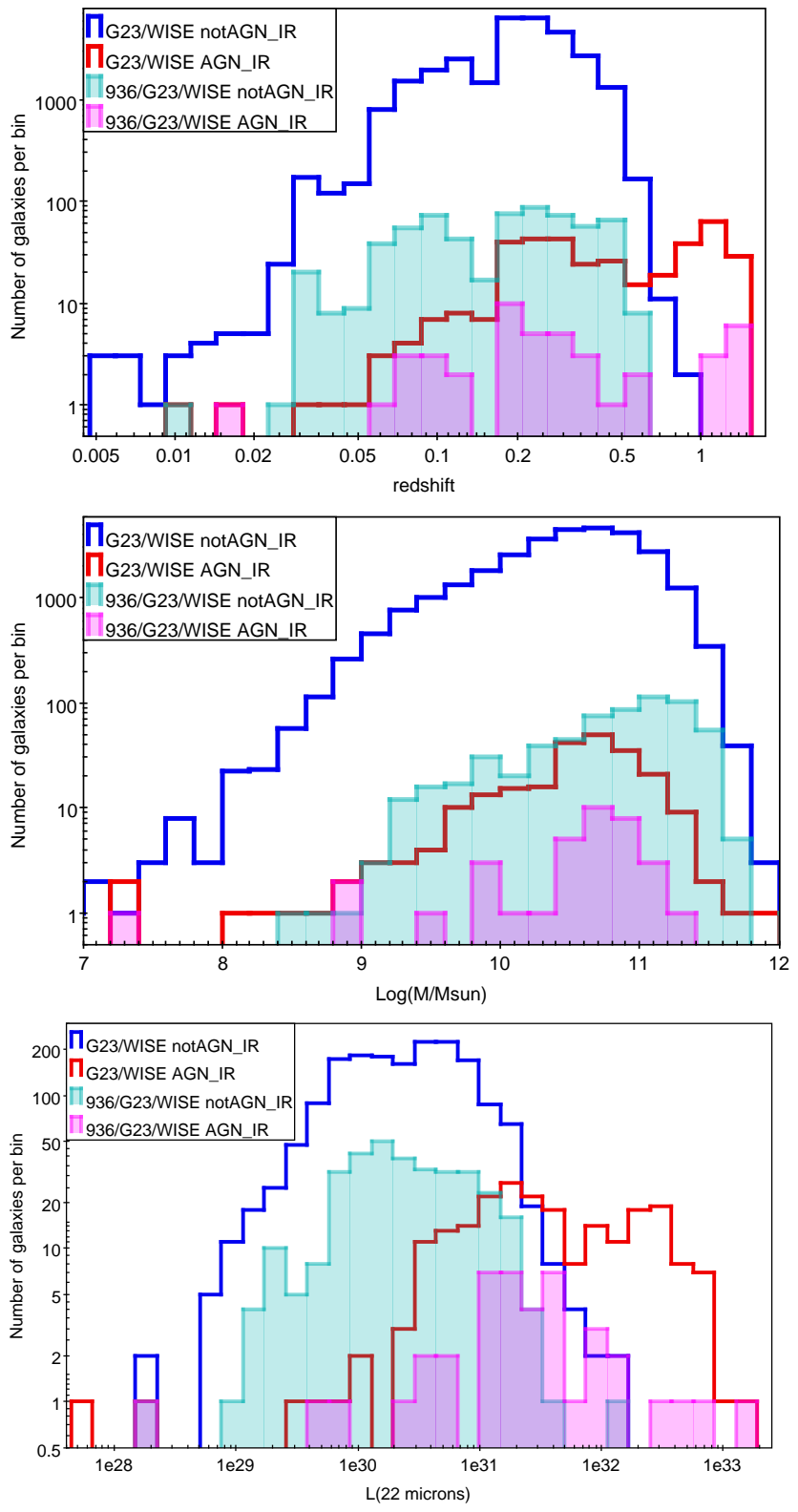

Figure 14. Comparison of the optical/IR galaxy sample with $936 \mathrm{MHz}$ emitting subsets. Shown are distributions of: redshift (top panel); mass (second panel); and $22 \mu m$ luminosity, $\mathrm{L}(22 \mu \mathrm{m})$, in $\mathrm{erg} / \mathrm{s} / \mathrm{Hz}$ (third panel, for subset with $\mathrm{w} 4 \mathrm{snr}>3$ ). Sets shown are G23/WISE notAGN ${ }_{I R}$ (blue), G23/WISE $\operatorname{AGN}_{I R}$ (red), 936/G23/WISE notAGN $I R$ (cyan) and 936/G23/WISE AGN $_{I R}$ (magenta).

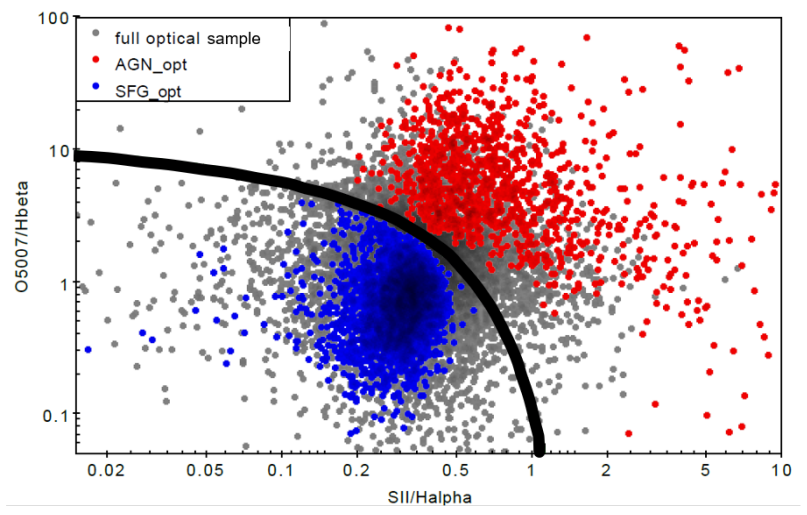

Figure 15. The BPT diagram, $[\mathrm{OIII}] \lambda 5007 / H \beta$ vs $[\mathrm{SII}] \lambda \lambda 6717,6731 / H \alpha$, for the set of G23 galaxies with all of the required lines in emission (grey). The dividing line between AGN and SFG (Kewley et al., 2001) is shown in black. The galaxies for which the error box lies entirely on the AGN side or on the SFG side of the dividing line are labelled $\mathrm{AGN}_{\text {opt }}$ (red) or $\mathrm{SFG}_{\text {opt }}$ (blue), respectively.
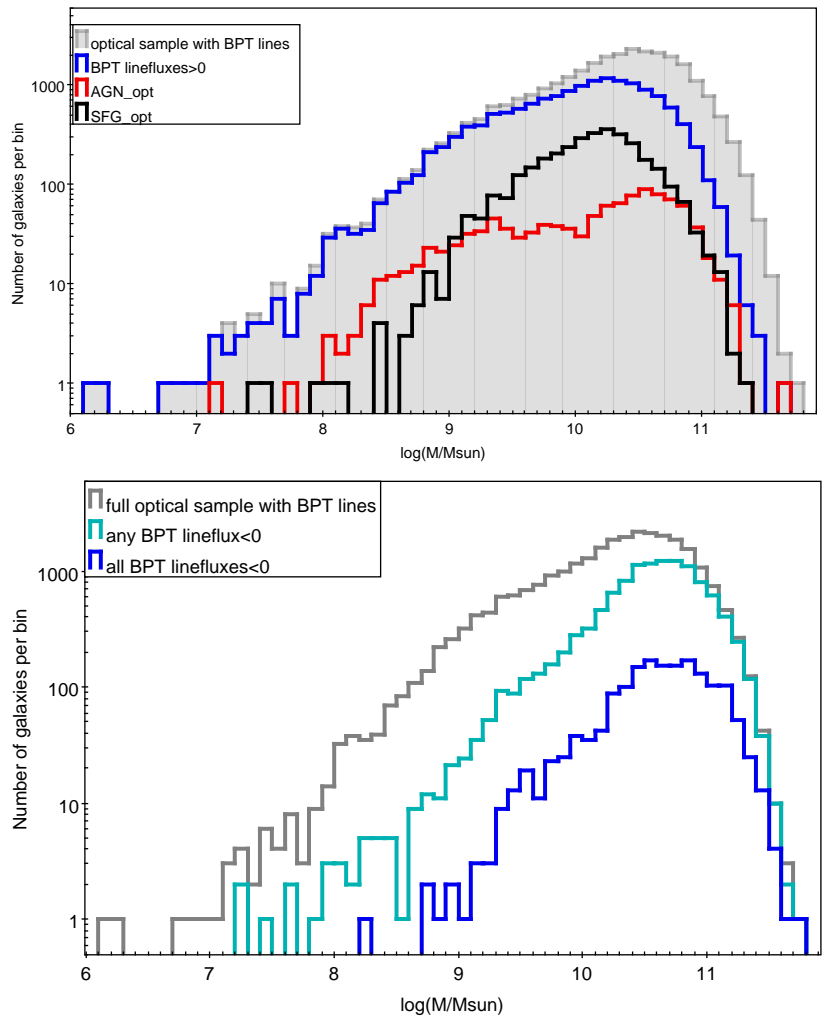

Figure 16. The distribution of masses (number of galaxies per mass bin, in units of $\left.\log \left(M / M_{\odot}\right)\right)$ for the different categories of G23 galaxies, all with measured BPT line fluxes. Top panel: the full sample (grey), galaxies with all 4 BPT lines in emission (blue), $\mathrm{SFG}_{I R}$ with BPT lines in emission (black), and $\mathrm{AGN}_{\text {opt }}$ with BPT lines in emission (red). Bottom panel: the full sample (grey), galaxies with at least one BPT line in absorption (cyan), and galaxies with all 4 BPT lines in absorption (blue). The top panel illustrates that galaxies with all BPT lines in emission have lowest mean mass, and $\mathrm{AGN}_{\text {opt }}$ have a broader, likely bimodal mass distribution. The bottom panel illustrates that galaxies with all BPT lines in absorption have highest mean mass. 

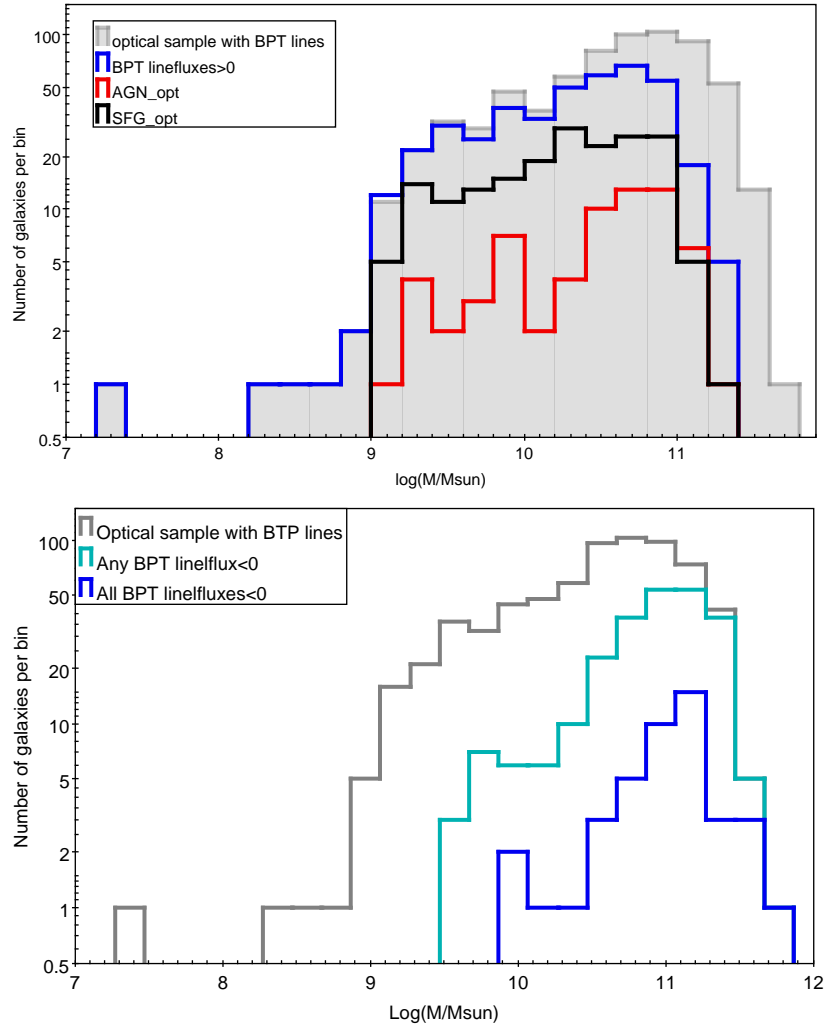

Figure 17. The distribution of masses (number of galaxies per mass bin, in units of $\left.\log \left(M / M_{\odot}\right)\right)$ for the different categories of G23 galaxies with $936 \mathrm{MHz}$ emission, all with measured BPT line fluxes. Top panel: the full radio/optical sample (grey), those with all 4 BPT lines in emission (blue), $\mathrm{SFG}_{\text {opt }}$ with BPT lines in emission (black), and $\mathrm{AGN}_{\text {opt }}$ with $\mathrm{BPT}$ lines in emission (red). Bottom panel: the full radio/optical sample (grey), those with at least one BPT line in absorption (cyan), and those with all 4 BPT lines in absorption (blue). The requirement of a $936 \mathrm{MHz}$ detection constrains the sample to relatively high masses, and the mass distributions of the optically classified AGN and SFGs with radio detections are similar.

$\mathrm{SFG}_{\text {opt }}$ by factor 1.35 .

Next, the BPT diagnostic was applied to the radio detected 936/G23 sample. Figure 17 shows the mass distributions for the 936/G23 galaxies and their five sub-categories. Comparison of the mass distributions in Figure 17 with those in Figure 16 shows that masses for galaxies with $936 \mathrm{MHz}$ emission are higher compared to galaxies without radio emission: $\mathrm{AGN}_{\text {opt }}$ with $936 \mathrm{MHz}$ emission has mean mass higher by factor 2.5 than the whole $\mathrm{AGN}_{\text {opt }}$ set; and $\mathrm{SFG}_{\text {opt }}$ with $936 \mathrm{MHz}$ emission has mean mass higher by factor 1.3 than the whole $\mathrm{SFG}_{\text {opt }}$ set. Similar results to that for $936 / \mathrm{G} 23$ are obtained when we apply the BPT diagnostic to 936/G23/WISE sample.

\subsubsection{Comparison of optical-classified and IR-classified samples}

The G23/WISE galaxies were separated into four categories: $\operatorname{not} \mathrm{AGN}_{I R} / \mathrm{SFG}_{\text {opt }}$; notAGN $\operatorname{Ag}_{I R} / \mathrm{AGN}_{\text {opt }}$;
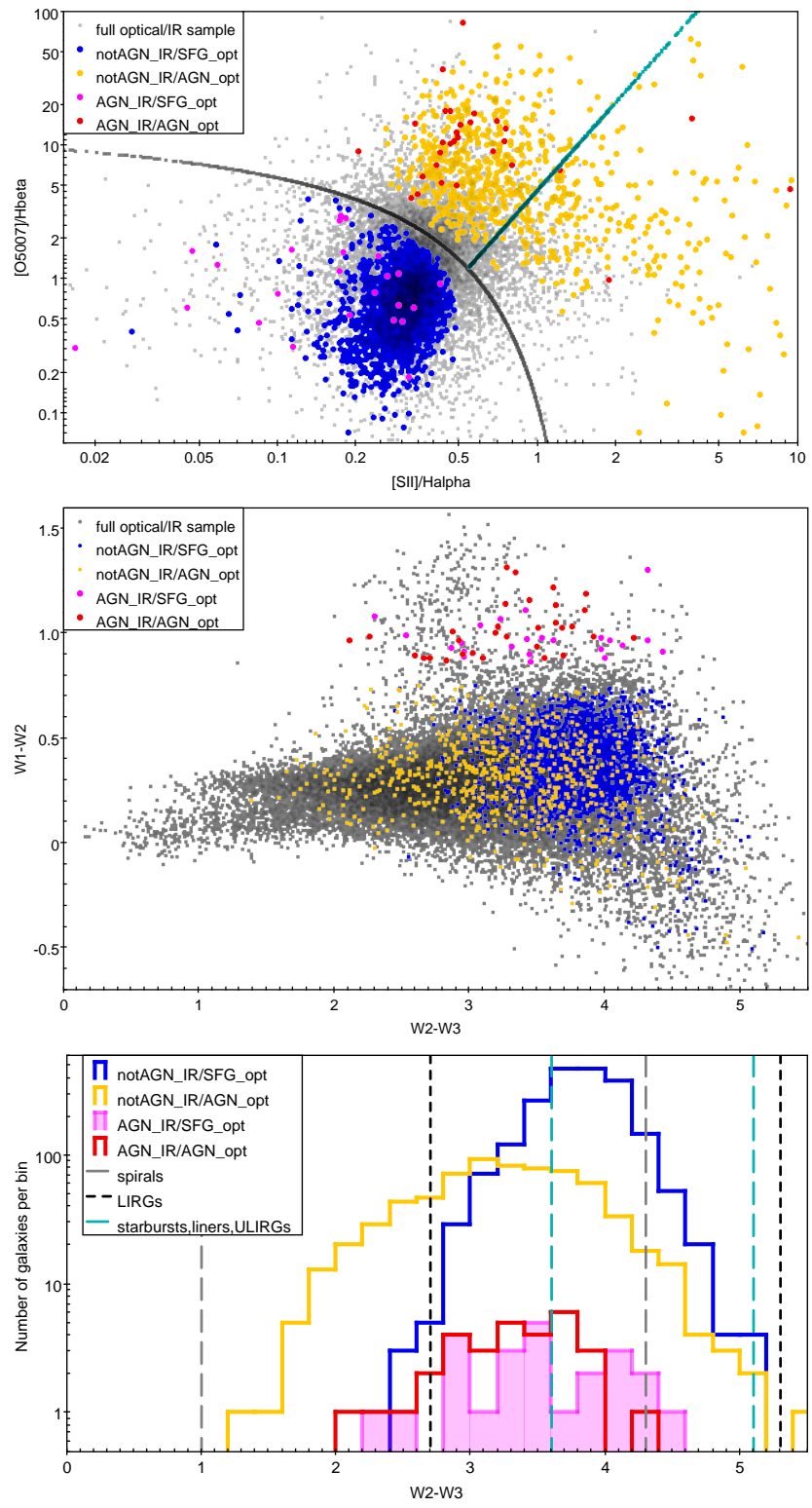

Figure 18. G23/WISE galaxies, classified as AGN or notAGN/SFG by the BPT and W1-W2 diagnostics. The full opti$\mathrm{cal} / \mathrm{IR}$ set of galaxies is shown in grey, notAGN $\mathrm{AR}_{I R}$ and $\mathrm{SFG}_{o p t}$ galaxies are marked in blue, galaxies classified as notAGN $I R$ and $\mathrm{AGN}_{\text {opt }}$ are marked in yellow, galaxies classified as $\mathrm{AGN}_{I R}$ and $\mathrm{SFG}_{\text {opt }}$ are marked in magenta, and those classified as $\mathrm{AGN}_{I R}$ and $\mathrm{AGN}_{\text {opt }}$ are marked in red. The top panel shows the BPT diagram, with the BPT line (Kewley et al., 2001) separating $\mathrm{SFG}_{\text {opt }}$ and $\mathrm{AGN}_{\text {opt }}$ in black; and with the line that marks the division (Sharp \& Bland-Hawthorn, 2010) between AGN-photo-ionized gas (upper-left of the cyan line) and shock-ionized gas (lowerright of the cyan line). The middle panel shows W1-W2 colour vs $\mathrm{W} 2-\mathrm{W} 3$ colour for sources with $\mathrm{W} 3 \mathrm{snr}>3$. The division between $\mathrm{AGN}_{I R}$ and notAGN $I R$ is at $\mathrm{W} 1-\mathrm{W} 2=0.8$. The bottom panel shows the distribution of W2-W3 colours for the different subsets (histograms) and the colour ranges (pairs of vertical dashed lines) for spirals, for LIRGs and for starbursts/liners/ULIRGs from Fig. 12 of Wright et al. (2010). 
$\mathrm{AGN}_{I R} / \mathrm{SFG}_{\text {opt }}$; and $\mathrm{AGN}_{I R} / \mathrm{AGN}_{\text {opt }}$. These are shown on a BPT diagram in the top panel of Figure 18. The $\mathrm{AGN}_{I R}$ and notAGN $I R$ galaxies are scattered on both sides of the line which forms the BPT criterion demonstrating that there is no clear correlation seen between the BPT and W1-W2 diagnostics.

The middle panel of Figure 18 shows the various classes of galaxies placed in the WISE colour-colour diagram. Wright et al. (2010) and Jarrett et al. (2011) showed that the notAGN ${ }_{I R}$ types include: ellipticals with $\mathrm{W} 2-\mathrm{W} 3$ of $\simeq 0.5-1.5$; spirals with $\mathrm{W} 2-\mathrm{W} 3$ of $\simeq 1.0-4.3$; luminous IR galaxies (LIRGs) with $\mathrm{W} 2$-W 3 of $\simeq 2.7-5.3$; and starbursts, liners and ultraluminous IR galaxies (ULIRGs) with W2-W3 of $\simeq 3.6-5.1$. The colour limits for these groups are shown in the bottom panel of Figure 18 overlaid on the distribution of W2-W3 colors. The notAGN $\mathrm{AR}_{I R} / \mathrm{SFG}_{\text {opt }}$ have a $\mathrm{W} 2$-W3 colour range consistent with that for LIRGs or a mixture of LIRGS and starbursts/liners/ULIRGs. The notAGN ${ }_{I R} / \mathrm{AGN}_{\text {opt }}$ have a wide $\mathrm{W} 2$-W3 colour range, consistent with a mixture of spirals and one or both of the other groups of galaxies (LIRGs and starbursts/liners/ULIRGs).

Table 5 shows that there is little quantitative correlation between the W1-W2 criterion and BPT criterion. Generally, the BPT classes of W1-W2 classified sources show poor correlation, e.g. for the G23/WISE sample, $\mathrm{AGN}_{I R}$ are $6.4 \%$ classified as $\mathrm{SFG}_{\text {opt }}$ and $8.2 \%$ classified as $\mathrm{AGN}_{\text {opt }}$. The W1-W2 classes of both $\mathrm{SFG}_{\text {opt }}$ and $\mathrm{AGN}_{\text {opt }}$ show most to be classified as notAGN $I R$.

The redshift distributions for the various categories of G23/WISE galaxies are shown in the left panels of Figure 19. The Kolmogorov-Smirnov test was applied and showed that the notAGN $\mathrm{AR}_{I R} / \mathrm{AGN}_{\text {opt }}$ subset has a different redshift distribution (lower redshifts) than the $\mathrm{AGN}_{I R} / \mathrm{SFG}_{\text {opt }}$ subset at $>99.9 \%$ confidence. The not $\mathrm{AGN}_{I R} / \mathrm{AGN}_{\text {opt }}$ have lower redshifts than not $\mathrm{AGN}_{I R} / \mathrm{SFG}_{\text {opt }}$ at the $99 \%$ confidence level, and the $\mathrm{AGN}_{I R} / \mathrm{AGN}_{\text {opt }}$ have lower redshifts than $\mathrm{AGN}_{I R} / \mathrm{SFG}_{\text {opt }}$ at only the $97.5 \%$ confidence level. The mass distributions for the various categories of G23/WISE galaxies are shown in the right panels of Figure 19.

We further split the G23/WISE GGN $_{\text {opt }}$ galaxies into subsets based on the dominant mechanism of ionization (Sharp \& Bland-Hawthorn, 2010): gas dominated by photo-ionization is left of the line (cyan line in the top panel of Figure 18) and shock-ionized gas is right of the line. Essentially the same line in the BPT diagram (Kewley et al., 2006) separates Seyferts (left of the line) from LINERs (right of the line). $64 \%$ of the $\mathrm{AGN}_{\text {opt }}$ galaxies are photo-ionized/Seyferts and $36 \%$ are shockionized/LINERs.

The redshift and mass distributions of the photoionized and shock-ionized subsets of $\mathrm{AGN}_{\text {opt }}$ galaxies are shown in Fig. 20. The $\mathrm{AGN}_{I R}$ subsets are small in number with 26 in the photo-ionized subset and 5 in the shock-ionized subset. Thus the redshift distributions cannot be distinguished. The $\mathrm{AGN}_{I R} /$ photo subset has a higher mean mass than the $\mathrm{AGN}_{I R}$ /shock subset, by a factor of $\sim 1.5$.

Comparison of the notAGN $\mathrm{AR}_{I R} /$ photo subset with the notAGN ${ }_{I R} /$ shock subset, shows that the shock-ionized fraction increases with redshift: from near 0 at $z<0.01$ to $\sim 50 \%$ for $z>0.15$. The not $\mathrm{AGN}_{I R} /$ shock subset has a narrower mass distribution than the photo-ionized subset and higher mean mass, by a factor of $\sim 1.6$. For the notAGN $\mathrm{AR}_{I R}$, the shock-ionized subsets are shifted to higher redshift and to higher mass compared to the photo-ionized subsets. Because of the correlation of mass with redshift (see Fig. 11), which is a selection effect of the galaxy survey, these are not independent effects.

\subsubsection{Comparison of optically-classified and $I R$-classified samples for the radio sources}

Table 4 lists basic properties of the our different samples of G23 galaxies, with additional selections based on detection by WISE, by $936 \mathrm{MHz}$ emission or by WISE W4 (22 $\mu \mathrm{m}$ band). The goal here is to determine how the radio emitters differ from their parent samples. The SFG radio emitters $\left(936 / \mathrm{G}_{23} / \mathrm{SFG}_{\text {opt }}\right)$ have lower mean redshift but similar mean mass, than the parent galaxy sample $\left(\mathrm{G} 23 / \mathrm{SFG}_{\text {opt }}\right)$. The $\mathrm{AGN}$ radio emitters (936/G23/AGN opt $\left._{\text {t }}\right)$ have lower mean redshift but higher mean mass, by factor 2.5 , than the parent galaxy sample $\left(\mathrm{G} 23 / \mathrm{AGN}_{\text {opt }}\right)$. The lower redshift can be understood as a selection effect, but the higher mass should be intrinsic.

The WISE selected radio and non-radio emitting samples, 936/G23/WISE and G23/WISE, are compared. $\mathrm{SFG}_{\text {opt }}$ and $\mathrm{AGN}_{\text {opt }}$ have lower redshift for the radio emitters, but the mean masses of $936 / \mathrm{G} 23 /$ WISE SFG and $\mathrm{AGN}_{\text {opt }}$ are essentially the same as for G23/WISE. With WISE selection, there are additional $\mathrm{AGN}_{I R}$ and notAGN $_{I R}$ categories. The radio emitters have lower redshift for $\mathrm{AGN}_{I R}$ and notAGN $\mathrm{AG}_{I R}$ than for the G23/WISE sample. The mean masses of radio emitting $\mathrm{AGN}_{I R}$ and the G23/WISE/AGN $I R$ are the same, but the radio emitting notAGN ${ }_{I R}$ have higher mean mass than G23/WISE/not $\mathrm{AGN}_{I R}$, by factor 2.1. The W1 magnitudes, related to the mass of the galaxy, of the radio emitters (936/G23/WISE) are brighter by $\sim 1$ magnitude for all 4 subsets than for G23/WISE.

The radio $(936 \mathrm{MHz})$ emitting subsets of $\mathrm{AGN}_{I R}$, notAGN ${ }_{I R}, \mathrm{SFG}_{\text {opt }}$ and $\mathrm{AGN}_{\text {opt }}$ sets are next compared. Table 4 lists some properties of these subsets, with the 936 label. First we consider the 936/G23/WISE sets. The highest redshift set is the $\mathrm{AGN}_{I R}$ subset. The notAGN $I R$ has highest mean mass (by a factor of $\sim 2-3$ over the other subsets). $\mathrm{AGN}_{\text {opt }}$ and $\mathrm{AGN}_{I R}$ are of similar mean masses and $\mathrm{SFG}_{\text {opt }}$ are of lowest mean mass. The mean $936 \mathrm{MHz}$ radio flux density of $\mathrm{AGN}_{I R}$ is much higher than any of the other subsets (by a factor of $\sim 9$ above the overall mean for 936/G23/WISE galaxies). 

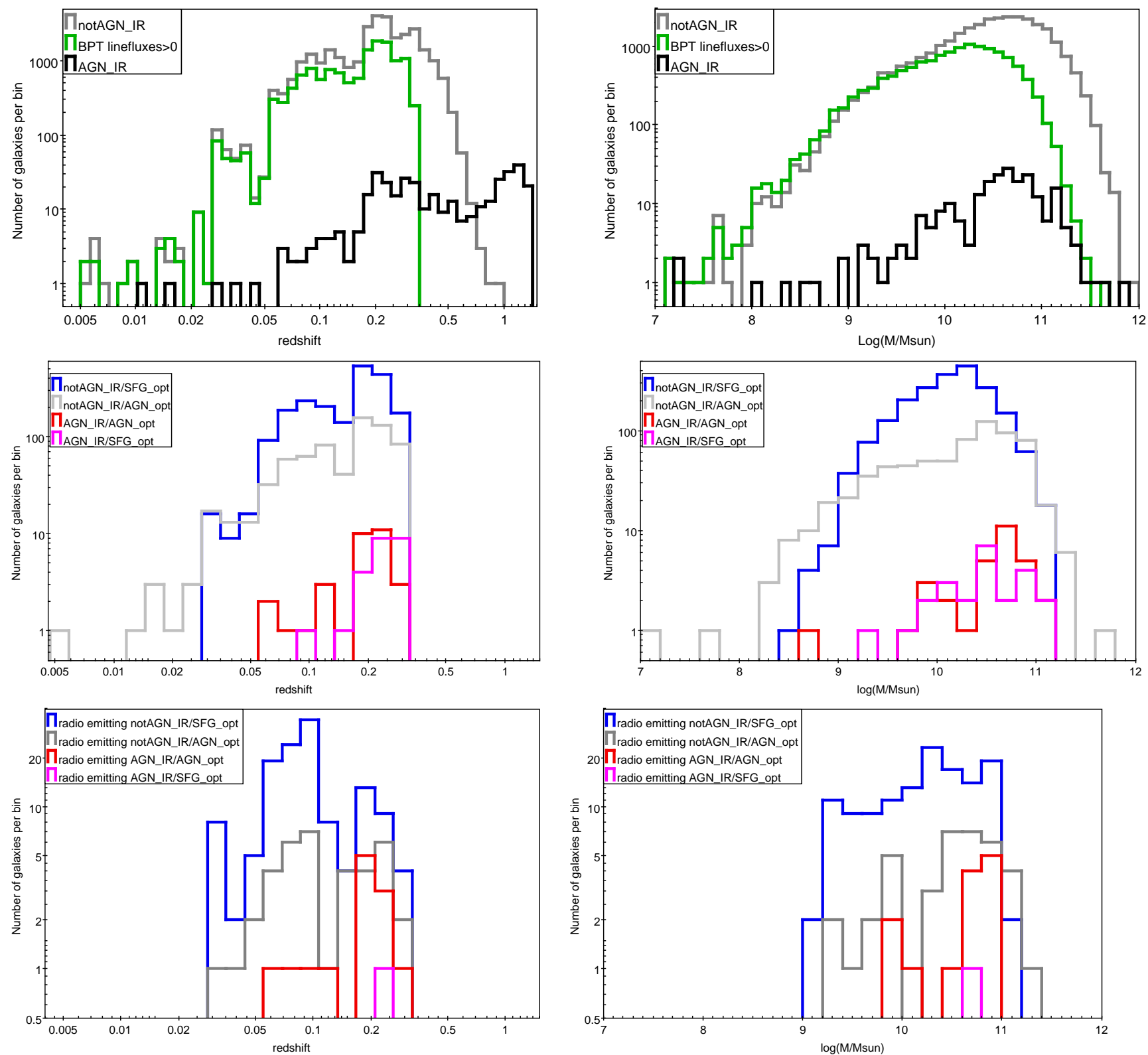

Figure 19. Distributions of redshift (number of galaxies per redshift bin, left panels) and distributions of mass (number of galaxies per mass bin, units of $\log \left(M / M_{\odot}\right)$, right panels) for the various subsets of G23/WISE galaxies. Subsets shown in the top two panels are notAGN $A_{I R}$ galaxies (grey), $\mathrm{AGN}_{I R}$ galaxies (black), and G23/WISE with all BPT lines in emission (green). Subsets shown in the middle and bottom panels are notAGN $I R / \mathrm{SFG}_{\text {opt }}$ (blue), notAGN $I R / \mathrm{AGN}_{\text {opt }}$ (grey), $\mathrm{AGN}_{I R} / \mathrm{AGN}_{\text {opt }}$ (red) and $\mathrm{AGN}_{I R} / \mathrm{SFG}_{\text {opt }}$ (magenta). 
Table 5 Numbers of AGN/notAGN/SFG using W1-W2 and BPT criteria, or of Emission-line Galaxies.

\begin{tabular}{|c|c|c|c|c|c|}
\hline Class $^{a}:$ & $\begin{array}{l}\operatorname{notAGN}_{I R} \\
\text { No. } / \%\end{array}$ & $\begin{array}{l}\mathrm{AGN}_{I R} \\
\text { No. } / \%\end{array}$ & $\begin{array}{l}\mathrm{SFG}_{o p t} \\
\text { No. } / \%\end{array}$ & $\begin{array}{l}\mathrm{AGN}_{\text {opt }} \\
\text { No. } / \%\end{array}$ & $\begin{array}{r}\text { Emission-line } \\
\text { No. } / \%\end{array}$ \\
\hline Sample & & & & & \\
\hline $\begin{array}{l}\text { G23/WISE } \\
\text { subgroups }\end{array}$ & $29983 / 95.3 \%$ & $374 / 1.2 \%$ & $2202 / 7.0 \%$ & $765 / 2.4 \%$ & $13149 / 41.8 \%$ \\
\hline $\operatorname{notAGN}_{I R}$ & $29983 / 100 \%$ & $0 / 0 \%$ & $2041 / 6.8 \%$ & $695 / 2.3 \%$ & $12121 / 40.4 \%$ \\
\hline $\mathrm{AGN}_{I R}$ & $0 / 0 \%$ & $374 / 100 \%$ & $24 / 6.4 \%$ & $31 / 8.2 \%$ & $108 / 28.9 \%$ \\
\hline $\mathrm{SFG}_{o p t}$ & $2014 / 92.7 \%$ & $24 / 1.1 \%$ & $2202 / 100 \%$ & $0 / 0 \%$ & $2202 / 100 \%$ \\
\hline $\mathrm{AGN}_{\text {opt }}$ & $695 / 90.8 \%$ & $31 / 4.1 \%$ & $0 / 0 \%$ & $765 / 100 \%$ & $765 / 100 \%$ \\
\hline $\begin{array}{l}\text { 936/G23/WISE } \\
\text { subgroups }\end{array}$ & $813 / 91.5 \%$ & $51 / 5.7 \%$ & $140 / 15.7 \%$ & $56 / 6.3 \%$ & $352 / 39.6 \%$ \\
\hline $\operatorname{notAGN}_{I R}$ & $813 / 100 \%$ & $0 / 0 \%$ & $130 / 16.0 \%$ & $38 / 4.7 \%$ & $308 / 37.9 \%$ \\
\hline $\mathrm{AGN}_{I R}$ & $0 / 0 \%$ & $51 / 100 \%$ & $1 / 2.0 \%$ & $13 / 25.4 \%$ & $26 / 51.0 \%$ \\
\hline $\mathrm{SFG}_{\text {opt }}$ & $130 / 92.9 \%$ & $1 / 0.7 \%$ & $140 / 100 \%$ & $0 / 0 \%$ & $140 / 100 \%$ \\
\hline $\mathrm{AGN}_{o p t}$ & $38 / 67.8 \%$ & $13 / 23.2 \%$ & $0 / 0 \%$ & $56 / 100 \%$ & $56 / 100 \%$ \\
\hline Class $^{b}:$ & $\begin{array}{c}\mathrm{AGN}_{I R} / \text { photo } \\
\text { No. }\end{array}$ & $\begin{array}{c}\text { notAGN }{ }_{I R} / \text { photo } \\
\text { No. }\end{array}$ & $\begin{array}{c}\mathrm{AGN}_{I R} / \text { shock } \\
\text { No. }\end{array}$ & $\begin{array}{c}\text { notAGN }{ }_{I R} / \text { shock } \\
\text { No. }\end{array}$ & \\
\hline G23/WISE AGN $_{\text {opt }}$ & 26 & 446 & 5 & 249 & \\
\hline
\end{tabular}
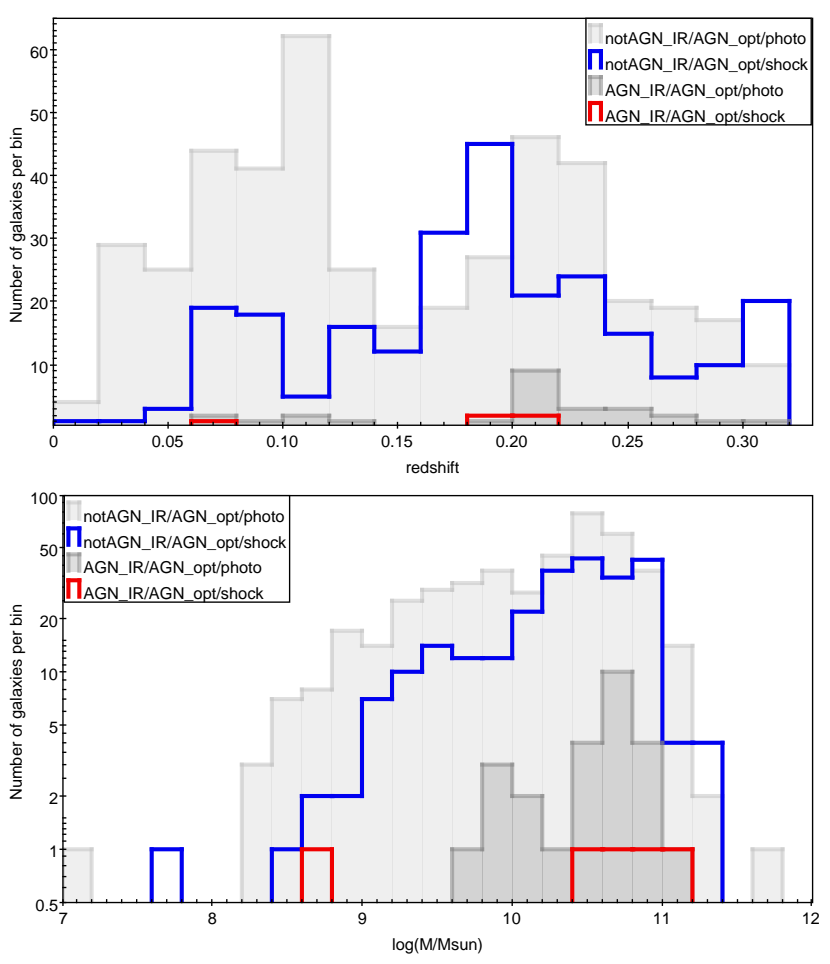

Figure 20. Distributions of redshift and mass for G23/WISE $\mathrm{AGN}_{\text {opt }}$ galaxies split into a subset dominated by photoionization ("/photo" in the label) and a subset dominated by shock ionization ("/shock" in the label). The sets shown are notAGN $I R / \mathrm{AGN}_{\text {opt }} /$ photo (light grey), notAGN $\mathrm{AR}_{\text {IR }} / \mathrm{AGN}_{\text {opt }} /$ shock (blue), $\mathrm{AGN}_{I R} / \mathrm{AGN}_{\text {opt }} /$ photo (dark grey) and $\mathrm{AGN}_{I R} / \mathrm{AGN}_{\text {opt }} /$ shock (red).
notAGN $\mathrm{AR}_{I R}$ have the second highest radio flux density, higher than either $\mathrm{AGN}_{\text {opt }}$ or $\mathrm{SFG}_{\text {opt }}$, by a factor of $\sim 2.5$.

396 of the 936/G23/WISE sources have W4 luminosity with $\mathrm{SN} \geq 3$ (labelled 936/G23/WISE w4snr $\geq 3$ ). The subsets' sample sizes are small except for notAGN ${ }_{I R}$. The $\mathrm{AGN}_{I R}$ are the most luminous subset at $936 \mathrm{MHz}$ and at $22 \mu \mathrm{m}$. The sub-categories, $\mathrm{AGN}_{I R} / \mathrm{AGN}_{\text {opt }}$, $\mathrm{AGN}_{I R} / \mathrm{SFG}_{\text {opt }}$ and $\mathrm{AGN}_{I R} /$ lineflux $<0$, are consistent with the same luminosity in the $22 \mu \mathrm{m}$ band, and the same flux density at $936 \mathrm{MHz}$.

Table 5 shows the breakdown of G23/WISE and 936/G23/WISE samples into IR AGN categories, BPT categories and the emission line galaxies category. The G23/WISE sample has a small fraction $(1.2 \%)$ classified as $\mathrm{AGN}_{I R}$ compared to not $\mathrm{AGN}_{I R}$. It has several percent classified as $\mathrm{SFG}_{o p t}$ or $\mathrm{AGN}_{o p t}$. For the radio emitting sample, 936/G23/WISE, the fraction of $\mathrm{AGN}_{I R}$ is $5.7 \%$, larger by factor $\sim 5$. The fraction of $\mathrm{SFG}_{o p t}$ or $\mathrm{AGN}_{\text {opt }}$ for $936 / \mathrm{G} 23 /$ WISE is larger by a factor of $\sim 2-2.5$ compared to G23/WISE. The rest of Table 5 is discussed in Section 4 below.

\section{DISCUSSION}

\subsection{Radio Source Distribution}

We compared flux density distributions of the single, double, and triple sources (Fig. 8), and found that the peak for doubles and triples occured at $\sim 20 \mathrm{mJy}$ compared to $\sim 1 \mathrm{mJy}$ for the singles. All of the doubles and triples (except one) are $\mathrm{AGN}_{I R}$. The single notAGN $\mathrm{AN}_{I R}$ 


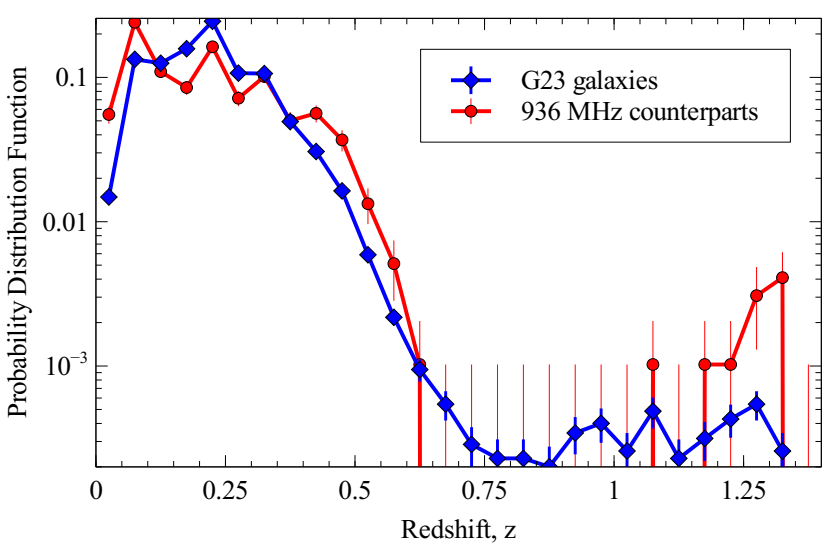

Figure 21. Redshift distributions $\left(P_{i}\right.$ for redshift bin $i$ ) for G23 galaxies and for $936 \mathrm{MHz}$ counterparts are shown by the blue and red curves. These are normalized by $\Sigma_{i} P_{i}=1$. There is an excess of radio counterparts for some redshift ranges and a deficit for other ranges. The $P_{i}$ for $1320 \mathrm{MHz}$ counterparts is almost identical with that for $936 \mathrm{MHz}$ counterparts, except that it has slightly larger error bars.

triple does not have a G23 optical galaxy counterpart or redshift. In contrast, the singles are dominated by the lower radio luminosity SFG (813 notAGN $\operatorname{As}_{I R}$ vs 51 $\left.\mathrm{AGN}_{I R}\right)$.

The redshift distributions of the G23 galaxies and those with $936 \mathrm{MHz}$ emission are shown in Figure 21. The radio emitters have similar redshifts to G23 galaxies, but they have real $(>3 \sigma)$ excesses at redshifts of $\simeq 0.0$ 0.10 and $0.35-0.60$ and a deficit at redshifts of $0.10-0.30$. There is a weak $(2 \sigma)$ excess for $z>1.2$. This may be a result from having multiple types of radio sources (SFG and AGN), each with a different spatial distribution. We find that the median redshifts for doubles and triples (which are all AGN) are 0.39 and 0.37 whilst the median redshift for single sources (which includes AGN and $\mathrm{SFG}$ ) is 0.21 .

Figures 11 and 12 illustrated that galaxies that are radio sources are more massive than average. The mean mass is higher by a factor of $\simeq 2.3$ and the peak mass of the distribution is shifted higher by a factor of $\simeq 3.2$.

We found K band and NUV magnitudes for G23 galaxies and radio counterparts by obtaining photometry from the G23 input catalogue. First we selected the set of G23 galaxies with photometry for the same sky area as the $936 \mathrm{MHz}$ map. We also selected the subset with photometry and spectra, to check for selection effects of requiring spectra. We then cross-matched the $936 \mathrm{MHz}$ sources to the G23 set and G23 subset with spectra to obtain photometry for the radio counterparts. Table 6 shows the means and standard deviations (SD) for the $\mathrm{K}$ band and NUV magnitudes of the galaxies and the radio counterparts. The mean $\mathrm{K}$ band flux for the radioemitting galaxies is higher by a factor of $\simeq 3$, consistent with the mass excess. The mean NUV band flux for the
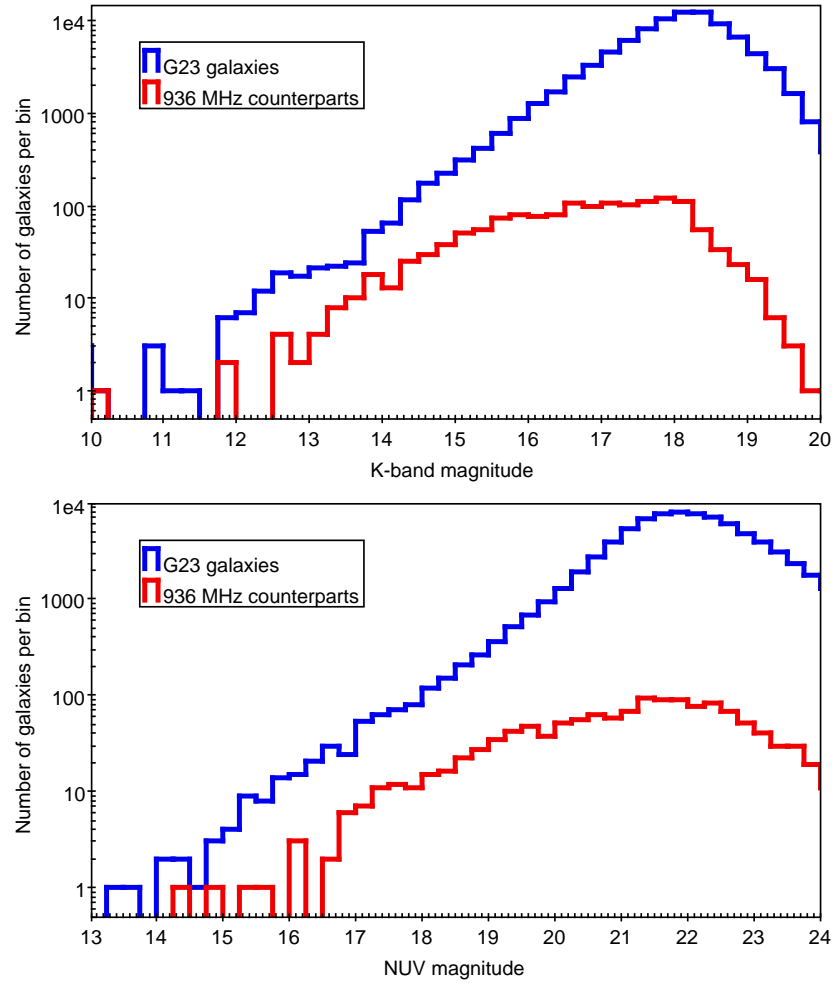

Figure 22. Top panel: K-band magnitude distributions for G23 galaxies with photometry (blue histogram) and for $936 \mathrm{MHz}$ counterparts (red histogram). Bottom panel: NUV magnitude distributions for G23 galaxies with photometry (blue histogram) and for $936 \mathrm{MHz}$ counterparts (red histogram). 
Table $6 \mathrm{~K}$ and NUV magnitudes of G23 galaxies and 936 $\mathrm{MHz}$ counterparts

\begin{tabular}{lccc}
\hline Sample & band & sample size $^{a}$ & $\begin{array}{c}\text { mean (SD) } \\
\text { (magnitude) }\end{array}$ \\
\hline G23ph $^{b}$ & $\mathrm{~K}$ & 92838 & $18.00(0.95)$ \\
& NUV & 81981 & $21.99(1.17)$ \\
G23ph $/ 936^{c}$ & $\mathrm{~K}$ & 1467 & $16.74(1.33)$ \\
& NUV & 1296 & $21.18(1.65)$ \\
\hline G23ph/sp & $\mathrm{K}$ & 35236 & $17.42(0.88)$ \\
& NUV & 32523 & $21.64(1.23)$ \\
G23ph/sp/936 & K & 930 & $16.33(1.12)$ \\
& NUV & 856 & $20.94(1.62)$ \\
\hline
\end{tabular}

a. Galaxies with measured $\mathrm{K}$ band magnitudes $(<21)$ and with measured NUV magnitudes $(<25)$ were selected for K and NUV samples, respectively.

b. G23ph is the set of G23 galaxies with photometry.

c. 936 indicates the sample is cross-matched with the $936 \mathrm{MHz}$ source catalogue using radius of $7^{\prime \prime}$.

d. G23ph/sp is the set of G23 galaxies with photometry and spectra.

radio emitters is higher by a factor of $\simeq 2$. The main effect of the additional requirement of having spectra (sp in Table 6) is the removal of the faint end of the magnitude distribution, hence reduction in the mean magnitudes by a small amount.

Figure 22 shows the distributions of K-band magnitudes and NUV magnitudes for the G23 galaxies with photometry and for the radio emitting subset. The radio emitters have much flatter distribution to low (brighter) magnitudes for both K and NUV. Radio-emission is detected much more frequently at high mass $\left(>10^{11} M_{\odot}\right)$ (Fig. 12), at bright $\mathrm{K}$ magnitude $(<15.5)$ and at bright NUV magnitude $(<19)$, by factors which range from $\sim 5-10$. Mass and $\mathrm{K}$ magnitude are expected to behave similarly because the mass is dominated by $\mathrm{K}$ and $\mathrm{M}$ stars (main-sequence and giant), and the optical light is dominated by $\mathrm{K}$ and $\mathrm{M}$ giants. The excess UV magnitude can come from active star formation or from the accretion disk associated with an AGN.

The fraction of galaxies that host a radio-loud AGN is known to rise strongly with stellar mass (Best et al., 2005 ), from nearly zero below stellar mass of $10^{10} M_{\odot}$ to $>30 \%$ for mass $>5 \times 10^{11} M_{\odot}$. For massive early-type galaxies, a strong correlation of radio power with K-band absolute magnitude has been demonstrated (e.g., Sadler et al., 1989; Fabbiano et al., 1989; Brown et al., 2011). For our full optical sample of galaxies (Figure 12), we find that there are no radio emitters below $2 \times 10^{8} M_{\odot}$ (with one exception). For the absorption line galaxies, which should be mostly massive early type galaxies, there are no radio emitters below $6 \times 10^{9} M_{\odot}$. This is consistent with the results of Brown et al. (2011) and Best et al. (2005), despite the difference in galaxy samples.

\subsection{AGN-SFG discrimination}

In Section 3.3.2, we used two tests to distinguish SFG from AGN: W1-W2 and BPT. Both tests have weaknesses. Two main types of AGN (for a review, see Best \& Heckman 2012) are High Excitation Radio Galaxies (HERGs) and Low Excitation Radio Galaxies (LERGs). The W1-W2 AGN diagnostic accepts some galaxies that are not AGN and misses AGN with weak mid-IR emission, such as LERGs. The BPT test can miss AGN with optically obscured nuclei and cannot be applied to the large fraction of galaxies that only present absorption lines.

Stern et al. (2012) showed that W1-W2 has high reliability using empirical AGN spectra and the galaxy spectral templates of Assef et al. (2010). However, that study did not consider all galaxy types. Some $\mathrm{AGN}_{I R}$ are not true AGN: Brown et al. (2014b) used observed spectra of nearby galaxies to show that observed spectral energy distributions of low metallicity blue compact dwarfs and extremely dusty lumininous IR galaxies (LIRGS) give W1-W2 colours like AGN. LIRGs are found predominantly in older, massive galaxies with strong stellar absorption lines, thus would be found among the $\sim 13000$ optical galaxies with absorption lines and not classified by the BPT criterion.

The WISE colors of LERGs and HERGs were investigated by Gürkan et al. (2014). They show that LERGs are found in the WISE color-colour diagram with W1$\mathrm{W} 2<0.8$ and $\mathrm{W} 2-\mathrm{W} 3$ in a broad range of $\sim 2-6$. The LERGs stand out most clearly from the other classes of AGN in the WISE $22 \mu \mathrm{m}$ vs radio $151 \mathrm{MHz}$ luminosity diagram (their Fig.8). We converted the boundaries of the regions containing HERGs and LERGs to $936 \mathrm{MHz}$ luminosity using a spectral index of -0.7 and plotted those boundaries on Figure 13. The HERG and LERG regions are distinct from the region occupied by SFGs. Most galaxies in our sample of radio emitting galaxies are located in a region consistent with SFGs, and few are consistent with HERGs or LERGs.

The $22 \mu m$ vs radio luminosity diagram was made for the subcategories of not $\mathrm{AGN}_{I R} / \mathrm{SFG}_{o p t}$, $\operatorname{notAGN}_{I R} / \mathrm{AGN}_{o p t}, \mathrm{AGN}_{I R} / \mathrm{AGN}_{\text {opt }}$ and $\mathrm{AGN}_{I R} /$ $\mathrm{SFG}_{\text {opt }}$. We find notAGN $I R / \mathrm{AGN}_{\text {opt }}$ and notAGN $I R /$ $\mathrm{SFG}_{\text {opt }}$ are not distinguishable in $22 \mu \mathrm{m}$ vs radio luminosity. The $\mathrm{AGN}_{I R} / \mathrm{AGN}_{\text {opt }}$ and $\mathrm{AGN}_{I R} / \mathrm{SFG}_{\text {opt }}$ are at higher $22 \mu \mathrm{m}$ and radio luminosity than the two notAGN ${ }_{I R}$ sets. However all 4 sets lie in the region of the blue points in the top panel of Figure $13^{4}$, and are in the region of SFGs rather than the regions of HERGs or LERGs.

The optical and IR classifications as AGN do not

\footnotetext{
${ }^{4}$ The requirement of optical classification (redshift $<0.32$ ) removes all of the high luminosity AGN in both $22 \mu \mathrm{m}$ and $926 \mathrm{MHz}$ bands. Fig. 13 does not include the optical (redshift) selection, so includes those high luminosity AGN.
} 
agree for a significant number of galaxies (see Table 5). 24 of the $\mathrm{SFG}_{\text {opt }}$ are $\mathrm{AGN}_{I R}$ (vs 2014 notAGN $\mathrm{AR}_{I R}$ ) and $\mathrm{AGN}_{\text {opt }}$ are mostly notAGN $\mathrm{AG}_{I R}$ (695) with few $\mathrm{AGN}_{I R}$ (31). What causes the difference between BPT and W1W2 classifications?

The W1-W2 classification is based on the slope of the 3.4 to $4.6 \mu \mathrm{m}$ IR spectrum. This is on the longwavelength Rayleigh-Jeans side of the stellar continuum peak for all stars, whether they are hot, massive and young, or old and cool. The presence of a strong AGN implies a significant contribution to the IR continuum by dust which is reradiating the AGN continuum. This results in a mid IR spectrum flatter than that seen in typical starbursts.

An AGN can have high extinction from the surrounding interstellar medium of the galaxy preventing the optical BPT lines from the AGN from being seen, while emission from star formation in the regions of the galaxy outside the high-extinction region may be detected. In this scenario the result is that such a galaxy will be classified as $\mathrm{SFG}_{\text {opt }}$, despite the presence of an AGN. Thus the $\mathrm{SFG}_{\text {opt }} / \mathrm{AGN}_{I R}$ galaxies can be explained as high-extinction AGN, accounting for one of the two conflicting classification pairs.

The extinction of galaxies is too small in the 3.4 to $4.6 \mu \mathrm{m}$ range to affect the spectral slope, so the W1W2 diagnostic can distinguish strong AGN from SFG. Faint AGN where the stellar continuum has a significant contribution ( $\gtrsim 50 \%$ ) in the 3.4 to $4.6 \mu \mathrm{m}$ range have W1-W2 $<0.8$ (Figure 2 of Stern et al. 2012), which results in faint AGN being classified as notAGN ${ }_{I R} / \mathrm{SFG}_{o p t}$ galaxies.

Wright et al. (2010) (their Figure 10) shows the WISE colour-colour diagram with different classes of galaxies in partially overlapping regions. The WISE colour-colour diagram for our sample (middle panel of Figure 18) shows that not $\mathrm{AGN}_{I R} / \mathrm{AGN}_{\text {opt }}$ galaxies are at smaller $\mathrm{W} 2-\mathrm{W} 3$ than notAGN $I R / \mathrm{SFG}_{\text {opt }}$ galaxies. Both of our sets overlap the spiral, LIRG and starburst regions. From the lower 2 panels of Fig. 18, we find that notAGN ${ }_{I R}$ galaxies with optical emission dominated by star formation $\left(\mathrm{SFG}_{\text {opt }}\right.$, blue histogram) are most consistent with W2-W3 colors of LIRGs. The notAGN $\mathrm{AR}_{I R} / \mathrm{AGN}_{\text {opt }}$ (yellow histogram) have W2-W3 colors consistent with mixture of spirals and LIRGs and possibly starbursts. The two $\mathrm{AGN}_{I R}$ categories $\left(\mathrm{SFG}_{\text {opt }}\right.$ and $\mathrm{AGN}_{\text {opt }}$ ) have few objects but spread across the LIRG, Seyfert, starburst, QSO and ULIRG regions of Figure 10 of Wright et al. (2010).

The notAGN $A_{I R} / \mathrm{AGN}_{\text {opt }}$ galaxies have optical lines showing AGN-like ionization, but their mid IR is powered mainly by stellar emission. The W1-W $2<0.8$ not $\mathrm{AGN}_{I R}$ criterion corresponds to the AGN contributing $<62 \%$ of the mid IR emission (Stern et al., 2012) for redshift $\mathrm{z}<0.32$ (the upper limit of galaxies classified by BPT). The left panel of Fig.2 of Stern et al. (2012) shows that almost the same limit $(<58 \%)$ applies for $\mathrm{E}(\mathrm{B}-\mathrm{V})=1$ and the doesn't change much for higher extinction if $\mathrm{z}<0.32$. Thus the AGN can contribute up to $60 \%$ to the mid IR emission, yet a galaxy will be classified as notAGN $I R$. We refer to this case as a weak AGN.

The notAGN ${ }_{I R} / \mathrm{AGN}_{\text {opt }}$ could be weak AGN where the stellar mid IR dominates over the AGN mid IR, but the AGN optical emission would have to dominate over the stellar optical emission to give the $\mathrm{AGN}_{\text {opt }}$ classification. Using the Kewley et al. (2006) criterion, we find $64 \%$ of $\mathrm{AGN}_{\text {opt }}$ are Seyferts and $36 \%$ are LINERs. Because the vast majority of both sets are notAGN ${ }_{I R}$, this implies both Seyferts and LINERs, with few exceptions, are not classified as AGN using IR colors.

Some fraction of AGN should be in the phase where the AGN has shut off but the AGN photoionization remains. Such AGN would be seen as $\mathrm{AGN}_{\text {opt }} /$ notAGN $_{I R} /$ photo, whereas active AGN would be seen as $\mathrm{AGN}_{\text {opt }} / \mathrm{AGN}_{I R} /$ photo. Table 5 gives the observed number of seen as these two types (446 and 26 , respectively). The ratio of these two types of $\sim 17$ in the current sample would imply the timescale for AGN photo-ionization to linger is greater than the AGN active phase by a factor of $\sim 17$. The estimated timescale for lingering photo-ionization in AGN is $10^{4}$ to $10^{5} \mathrm{yr}$ (Schawinski et al., 2015), and the AGN active timescale is estimated at $10^{5} \mathrm{yr}$. Thus the expected ratio of $\mathrm{AGN}_{\text {opt }} /$ notAGN $\mathrm{AR}_{I R} /$ photo to $\mathrm{AGN}_{\text {opt }} / \mathrm{AGN}_{I R} /$ photo should be $\sim 0.1$ to 1 , rather than $\sim 17$. So, either: a) only $\sim 3$ to 30 of the $446 \mathrm{AGN}_{\text {opt }} /$ notAGN $\mathrm{AG}_{I R} /$ photo can be explained by lingering photo-ionization; or b) the AGN on and off timescales are inaccurate by 1 to 2 orders of magnitude. In the first case, there must be another explanation for most of the $\mathrm{AGN}_{\text {opt }} / \operatorname{not} \mathrm{AGN}_{I R} /$ photo.

In summary, there is the explanation of extinction for the apparent conflicting classification $\mathrm{AGN}_{I R} / \mathrm{SFG}_{\text {opt }}$, but no single convincing explanation for notAGN $\mathrm{AR}_{I R} / \mathrm{AGN}_{\text {opt }}$.

\section{SUMMARY AND CONCLUSION}

We have processed ASKAP commissioning observations of the GAMA G23 field in two frequency bands centred at $936 \mathrm{MHz}$ and $1320 \mathrm{MHz}$, and have demonstrated that ASKAP produces excellent image quality, allowing identification of radio sources down to $\sim 1$ mJy with positions accurate to $\sim 5^{\prime \prime}$. We extracted the radio components and identified multiple component radio sources to produce $936 \mathrm{MHz}$ and $1320 \mathrm{MHz}$ radio source catalogues.

The radio sources were position matched to GAMA G23 galaxies, to WISE IR sources and to G23 galaxies with WISE IR counterparts. The masses for the G23 galaxies were based on masses of photometry-matched galaxies from the GAMA equatorial fields. A summary of our analysis of the multi-wavelength data set is as 
follows.

- Of the $\sim 5800$ radio sources, $\sim 1000$ have counterparts amongst the G23 galaxies, 3000 have WISE IR counterparts and $\sim 900$ have both G23 galaxy and WISE counterparts. Some fraction of the radio sources without WISE counterparts will be AGN too faint to be detected by WISE (IR-faint radio sources; e.g. Collier et al., 2014). The G23 galaxy radio sources tend to be those at the high-mass end $\left(\gtrsim 10^{10} \mathrm{M}_{\odot}\right.$ ) of the galaxy distribution (top panel of Figure 11).

- Galaxies that are detected in radio are observed to be more massive, and brighter at K-band and UV, than those without radio sources. Radio emission from AGN is expected to be brighter for more massive galaxies, which are brighter in K band. Similarly, radio emission from star formation and UV emission are stronger for galaxies with active star formation.

- The majority of the ASKAP radio sources show the well-known correlation (Rieke et al., 2009) for SFG between the radio and mid-IR luminosities (Figure 13). A few tens of sources have excess radio emission, and are likely to host AGN, although not all are classified as AGN by their IR colour.

- The G23 galaxies were classified as $\mathrm{AGN}_{I R}$ or notAGN ${ }_{I R}$, based on the W1-W2 indicator. $\sim 380$ galaxies are classified as $\mathrm{AGN}_{I R}$, compared to $\sim 30,000$ classified as notAGN $A_{I R} . \mathrm{AGN}_{I R}$ have higher redshifts than notAGN ${ }_{I R}$ (factor $\sim 3$ ) but similar masses. $\mathrm{AGN}_{I R}$ have much higher $22 \mu \mathrm{m}$ luminosities (factor $\sim 30$ ). Only $\sim 50$ of these $\mathrm{AGN}_{I R}$ are radio sources.

- Using the BPT diagnostic, galaxies were classified as $\mathrm{AGN}_{\text {opt }}$ or $\mathrm{SFG}_{\text {opt }}$. 770 galaxies are classified as $\mathrm{AGN}_{\text {opt }}$ and $\sim 2200$ classified as $\mathrm{SFG}_{\text {opt }}$. $\mathrm{AGN}_{\text {opt }}$ have similar redshifts to $\mathrm{SFG}_{\text {opt }}$ but a broader mass distribution.

- We find disagreement between these two AGN indicators. 24 of $55 \mathrm{AGN}_{I R}$ are classified as $\mathrm{SFG}_{\text {opt }}$ and $\sim 700$ of 2800 notAGN $A_{I R}$ are classified as $\mathrm{AGN}_{\text {opt }}$ (Table 5). The $\mathrm{AGN}_{I R} / \mathrm{SFG}_{\text {opt }}$ galaxies can be explained in terms of obscured $\mathrm{AGN}_{I R}$. The reasons why $\mathrm{AGN}_{o p t}$ are classified as notAGN $I R$ are not clearly understood yet.

- The radio sources were separated into $\mathrm{AGN}_{I R}$, notAGN $A_{I R}, \mathrm{AGN}_{\text {opt }}$ and $\mathrm{SFG}_{\text {opt }}$ subsets. These radio subsets are smaller by $\sim 1$ order of magnitude than the galaxy subsets. Each of the 4 radio subsets has indistinguishable redshift distribution from that of the corresponding parent galaxy subset (Figure 19, left panels). We find that the level of disagreement between optical and IR AGN diagnostics is similar for the radio emitting subsets as for the corresponding parent galaxy subset (Table 5).
The results presented in this paper are a first step towards combining large deep radio survey data with large well-studied samples of galaxies in the nearby Universe with extensive multi-wavelength data. The EMU survey will be almost an order of magnitude more sensitive than the observations presented here, and this, combined with even deeper optical and IR observations, will provide even greater overlap between the radio, optical, and IR samples of galaxies, enabling radio observations to become another tool that will be used routinely to characterise and understand galaxies in the local Universe.

\section{ACKNOWLEDGEMENTS}

The Australian SKA Pathfinder is part of the Australia Telescope National Facility which is managed by CSIRO. Operation of ASKAP is funded by the Australian Government with support from the National Collaborative Research Infrastructure Strategy. ASKAP uses the resources of the Pawsey Supercomputing Centre. Establishment of ASKAP, the Murchison Radio-astronomy Observatory and the Pawsey Supercomputing Centre are initiatives of the Australian Government, with support from the Government of Western Australia and the Science and Industry Endowment Fund. We acknowledge the Wajarri Yamatji people as the traditional owners of the Observatory site. GAMA is a joint EuropeanAustralasian project based around a spectroscopic campaign using the Anglo-Australian Telescope. The GAMA input catalogue is based on data taken from the Sloan Digital Sky Survey and the UKIRT Infrared Deep Sky Survey. Complementary imaging of the GAMA regions is being obtained by a number of independent survey programmes including GALEX MIS, VST KiDS, VISTA VIKING, WISE, Herschel-ATLAS, GMRT and ASKAP providing UV to radio coverage. GAMA is funded by the STFC (UK), the ARC (Australia), the AAO, and the participating institutions. The GAMA website is http://www.gama-survey.org/ . This publication makes use of data products from the Wide-field Infrared Survey Explorer, which is a joint project of the University of California, Los Angeles, and the Jet Propulsion Laboratory/California Institute of Technology, funded by the National Aeronautics and Space Administration. DL is supported by a grant from the Natural Sciences and Engineering Research Council of Canada. The referee is acknowledged for a number of suggestions that led to improvements in this paper.

\section{REFERENCES}

Assef R. J., et al., 2010, ApJ, 713, 970

Baldwin J. A., Phillips M. M., Terlevich R., 1981, PASP, 93, 5

Best P. N., Heckman T. M., 2012, MNRAS, 421, 1569 
Best P. N., Kauffmann G., Heckman T. M., Brinchmann J., Charlot S., Ivezić Ž., White S. D. M., 2005, MNRAS, 362, 25

Bock D. C.-J., Large M. I., Sadler E. M., 1999, AJ, 117, 1578

Brown M. J. I., Jannuzi B. T., Floyd D. J. E., Mould J. R., 2011, ApJ, 731, L41

Brown M. J. I., Jarrett T. H., Cluver M. E., 2014a, PASA, 31, HASH

Brown M. J. I., et al., 2014b, ApJS, 212, 18

Brown M. J. I., et al., 2017, ApJ, 847, 136

Chabrier G., 2003, PASP, 115, 763

Cluver M. E., et al., 2014, ApJ, 782, 90

Cluver M. E., Jarrett T. H., Dale D. A., Smith J.-D. T., August T., Brown M. J. I., 2017, ApJ, 850, 68

Collier J. D., et al., 2014, MNRAS, 439, 545

Condon J. J., Cotton W. D., Greisen E. W., Yin Q. F., Perley R. A., Taylor G. B., Broderick J. J., 1998, AJ, 115,1693

Cornwell T. J., Voronkov M. A., Humphreys B., 2012, in Image Reconstruction from Incomplete Data VII. p. 85000L (arXiv:1207.5861), doi:10.1117/12.929336

Driver S. P., et al., 2011, MNRAS, 413, 971

Driver S. P., et al., 2016, MNRAS, 455, 3911

Edge A., Sutherland W., Kuijken K., Driver S., McMahon R., Eales S., Emerson J. P., 2013, The Messenger, 154,32

Fabbiano G., Gioia I. M., Trinchieri G., 1989, ApJ, 347, 127

Gürkan G., Hardcastle M. J., Jarvis M. J., 2014, MNRAS, 438, 1149

Hancock P. J., Murphy T., Gaensler B. M., Hopkins A., Curran J. R., 2012, Aegean: Compact source finding in radio images, Astrophysics Source Code Library (ascl:1212.009)

Heckman T. M., Best P. N., 2014, ARA\&A, 52, 589

Hopkins A. M., Afonso J., Chan B., Cram L. E., Georgakakis A., Mobasher B., 2003, AJ, 125, 465

Hopkins A. M., et al., 2013, MNRAS, 430, 2047

Jarrett T. H., et al., 2011, ApJ, 735, 112

Johnston S., et al., 2008, Experimental Astronomy, 22, 151

Kennicutt Jr. R. C., et al., 2009, ApJ, 703, 1672

Kewley L. J., Dopita M. A., Sutherland R. S., Heisler C. A., Trevena J., 2001, ApJ, 556, 121

Kewley L. J., Groves B., Kauffmann G., Heckman T., 2006, MNRAS, 372, 961

Liske J., et al., 2015, MNRAS, 452, 2087

Magliocchetti M., Maddox S. J., Lahav O., Wall J. V., 1998, MNRAS, 300, 257

McConnell D., et al., 2016, PASA, 33, e042

Norris R. P., 2017, Nature Astronomy, 1, 671

Norris R. P., et al., 2006, AJ, 132, 2409
Norris R. P., et al., 2011, PASA, 28, 215

Norris R. P., et al., 2013, PASA, 30, e020

Reynolds J., 1994, Technical report, ATNF Technical Memos 39.3 040. Australia Telescope National Facility

Ricci F., Marchesi S., Shankar F., La Franca F., Civano F., 2017, MNRAS, 465, 1915

Rieke G. H., Alonso-Herrero A., Weiner B. J., PérezGonzález P. G., Blaylock M., Donley J. L., Marcillac D., 2009, ApJ, 692, 556

Sadler E. M., Jenkins C. R., Kotanyi C. G., 1989, MNRAS, 240, 591

Schawinski K., Koss M., Berney S., Sartori L. F., 2015, MNRAS, 451, 2517

Schinckel A. E., Bunton J. D., Cornwell T. J., Feain I., Hay S. G., 2012, in Ground-based and Airborne Telescopes IV. p. 84442A, doi:10.1117/12.926959

Seymour N., et al., 2008, MNRAS, 386, 1695

Sharp R. G., Bland-Hawthorn J., 2010, ApJ, 711, 818

Stern D., et al., 2012, ApJ, 753, 30

Taylor M., 2011, TOPCAT: Tool for OPerations on Catalogues And Tables, Astrophysics Source Code Library (ascl:1101.010)

Taylor E. N., et al., 2011, MNRAS, 418, 1587

Wolf C., et al., 2017, MNRAS, 466, 1582

Wright E. L., et al., 2010, AJ, 140, 1868

de Jong J. T. A., Verdoes Kleijn G. A., Kuijken K. H., Valentijn E. A., 2013, Experimental Astronomy, 35, 25 